\title{
Analysis of Cylindrical Cavity Expansion in Anisotropic Critical State Soils Under Drained Conditions
}

\begin{tabular}{|c|c|}
\hline Journal: & Canadian Geotechnical Journal \\
\hline Manuscript ID & cgj-2018-0025.R1 \\
\hline Manuscript Type: & Article \\
\hline $\begin{array}{r}\text { Date Submitted by the } \\
\text { Author: }\end{array}$ & 12-Jul-2018 \\
\hline Complete List of Authors: & $\begin{array}{l}\text { Liu, Kai; Louisiana State University College of Engineering, } \\
\text { Department of Civil \& Environmental Engineering } \\
\text { Chen, Shengli; Louisiana State University System, Department } \\
\text { of Civil \& Environmental Engineering }\end{array}$ \\
\hline $\begin{array}{r}\text { Is the invited manuscript } \\
\text { for consideration in a } \\
\text { Special Issue? : }\end{array}$ & Not applicable (regular submission) \\
\hline Keyword: & $\begin{array}{l}\text { anisotropic Cam Clay model, cylindrical cavity expansion, } \\
\text { drained condition, semi-analytical solution, stress path }\end{array}$ \\
\hline
\end{tabular}




\title{
ANALYSIS OF CYLINDRICAL CAVITY EXPANSION IN ANISOTROPIC CRITICAl State SoILS UNDER DRAINed CONDITIONS
}

\author{
K. Liu, and S. L. Chen
}

K. Liu, Department of Civil \& Environmental Engineering, Louisiana State University, Baton Rouge, LA, USA. Email: kliu21@1su.edu

S. L. Chen, Department of Civil \& Environmental Engineering, Louisiana State University, Baton Rouge, LA, USA. Email: shenglichen@lsu.edu 
Abstract: This paper presents a semi-analytical solution for the drained cylindrical cavity expansion problem using the well-known anisotropic modified Cam Clay model proposed by Dafalias (1987). The prominent feature of this elastoplastic model, i.e., its capability to describe both the initial fabric anisotropy and stress-induced anisotropy of soils, makes the anisotropic elastoplastic solution derived herein for the cavity problem a more realistic one. Following the novel solution scheme developed by Chen and Abousleiman (2013) that links between the Eulerian and Lagrangian formulations of the condition of radial equilibrium, the plastic zone solution can be eventually obtained by solving a system of eight partial differential equations with the three stress components, three anisotropic hardening parameters, specific volume, and preconsolidation pressure being the basic unknowns. Parametric studies have then been conducted to explore the influences of $K_{0}$ consolidation anisotropy and overconsolidation ratio $(O C R)$, and their pronounced impacts on the stress patterns outside the cavity as well as on the development of stress-induced anisotropy are clearly observed.

Keywords: anisotropic Cam Clay model; cylindrical cavity expansion; drained condition; semianalytical solution; stress path 


\section{Introduction}

Cylindrical cavity expansion, one of the basic boundary value problems in theoretical geomechanics and geotechnical engineering (Yu 2000), has received extensive attention over the past few decades (Gibson and Anderson 1961; Vesic 1972; Collins and Yu 1996; Ladanyi and Foriero 1998; Cudmani and Osinov 2001; Chen and Abousleiman 2016), due to its versatile applications in the prediction of driven pile capacity and in the interpretation of pressuremeter and piezocone penetration tests. Much current research is aimed at the development of new/exact semi-analytical solution techniques (Chen and Abousleiman 2012, 2013) and the adoption of more realistic/sophisticated elastoplastic models for dealing with such problems (Chen and Abousleiman 2016, 2018; Li et al. 2016; Mo and Yu 2017a, 2017b; Sivasithamparam and Castro 2018).

Vesic (1972) appeared to be the first one to solve the cylindrical/spherical cavity expansion problems under both drained and undrained conditions using Mohr-Coulomb failure criterion, some approximations/simplifications had been made in the derivation of solutions though. Carter el al. (1986) investigated the expansion of cylindrical/spherical cavities in an ideal, cohesive frictional soil subjected to fully isotropic in situ stresses, and presented the pressure-expansion relation in a closed-form manner. Starting with the development of shear strain accumulated during the process of undrained expansion, Collins and Yu (1996) later proposed a theoretical framework that leads to analytical and semi-analytical solutions for cavity expanded in both original and modified Cam Clay soils. More recently, two novel solution methodologies have been developed by Chen and Abousleiman (2012, 2013), which do not require any approximations to be made about the mean and deviatoric stresses to solve the undrained and drained cylindrical cavity expansion problems in modified Cam Clay soil. The same authors 
subsequently expanded upon their solution techniques, by applying the more advanced model of bounding surface plasticity, to tackle the drained/undrained cavity contraction problems with applications in tunnelling and wellbore drilling (Chen and Abousleiman 2016).

Given the fact that natural clayey soils are inevitably anisotropic due to the deposition process and subsequent loading history (Tavernas and Leroueil 1977; Banerjee and Yousif 1986; Voyiadjis and Song 2000; Wheeler et al. 2003), some cavity expansion solutions on account of the mechanical anisotropy of soils recently have also been developed, though quite limited. For example, Kolymbas et al. (2012) analyzed the cylindrical cavity expansion problem in an analytical fashion based on the assumption of cross-anisotropic linear elastic material, and then applied the obtained approximate solution to back calculate the anisotropic parameters of rock mass surrounding the tunnel Mais. Li et al. (2016, 2017), on the other hand, following the methods proposed by Chen and Abousleiman (2012, 2013), developed semi-analytical solutions for the undrained and drained cavity expansion problems in $K_{0}$ consolidated, anisotropic elastoplastic soils, but the rotation of the yield surface was deemed to be relevant merely to the inherent sedimentation anisotropy and be fixed in the stress space. This limitation has been overcome in a most recent paper by Chen and Liu (2018), who offered a rigorous cavity expansion solution under undrained condition with the due consideration of both initial deposition and stress-induced anisotropy, using the well-known rotational hardening elastoplastic model proposed by Dafalias (1987). It is worthy to mention that a similar undrained solution was also simultaneously and independently derived by Sivasithamparam and Castro (2018), where a different anisotropic elastoplastic model S-CLAY1 (Wheeler et al. 2003) incorporating both plastic volumetric strain and plastic shear strain within the rotational hardening rule has been employed in their formulations. 
The present paper continues on the theme of developing analytical solutions for cavity expansion in elastoplastic soils, this time with an extension of the work of Chen and Liu (2018) from undrained to drained loading conditions. The derivation presented in this work is again based on Dafalias' (1987) representative anisotropic Cam Clay model and on the leverage of the solution procedure originally proposed by Chen and Abousleiman (2013), which enables an innovative conversion of the radial equilibrium equation between Eulerian and Lagrangian forms. It is found that the plastic zone solution can be eventually obtained by solving a system of eight partial differential equations with the three stress components, three anisotropic hardening parameters, specific volume, and preconsolidation pressure being the basic unknowns. Intensive parametric studies have been conducted firstly to validate the present anisotropic solutions and check its accuracy, and then to investigate the influences of $K_{0}$ consolidation anisotropy and of the overconsolidation ratio $(O C R)$. The numerical results clearly demonstrate the significant impacts of these two key parameters on the stress patterns outside the cavity and the development of stress-induced anisotropy as well. The proposed analytical solution provides a useful benchmark from which the numerical results may be evaluated for various geotechnical boundary value problems involving the sophisticated anisotropic critical state plasticity models.

\section{Problem statement and assumptions}

Consider a cylindrical cavity with initial radius of $a_{0}$, being expanded in a fully saturated anisotropic modified Cam Clay soil (Dafalias 1987) whose in situ stresses are, respectively, $\sigma_{h}$ in the horizontal plane and $\sigma_{v}$ in the vertical direction, as shown in Fig. 1. Note that for the drained expansion problem, all the stresses are pertaining to the effective stresses (Chen and Abousleimna 2013). As is usually done in the previous studies, the deformation of the soil surrounding the cavity is assumed to be in conditions of axial symmetry and plane strain and, 
therefore, the problem actually becomes one-dimensional.

When the internal pressure is increased from its initial value $\sigma_{a 0}$ (equal to $\sigma_{h}$ ) to the current one of $\sigma_{a}$, the cavity concurrently will be expanded to a larger radius of $a$. At this particular moment a generic soil particle initially at $r_{x 0}$ has moved outward to a new position of $r_{x}$, and the corresponding position of the elastic-plastic interface is denoted by $r_{p}$, which was originally located at $r_{p 0}$. Fig. 1 illustrates the scenario that involves only a plastic deformation annulus $a \leq r \leq r_{p}$ and an external, purely elastic region beyond the radius $r=r_{p}$. However, if the cavity is further and sufficiently expanded, one may expect that a third critical state region would be formed immediately outside the cavity, encompassed by the above two regions of elastic and plastic deformations.

As in Chen and Liu (2018), to simplify the mathematical derivation so as to obtain the semianalytical elastoplastic solution, no attempt will be made in the present work to incorporate the elastic anisotropy of soil behaviour. Nevertheless, it is supposed herein that the shear modulus $G$ and Young's modulus $E$ are both dependent on the mean stress $p$ and specific volume $v$ within the critical state soil mechanics framework (Schofield and Wroth, 1968), and take the following form

$$
G=\frac{3(1-2 \mu) v p}{2(1+\mu) \kappa}, \quad E=2(1+\mu) G
$$

where $\mu$ is Poisson's ratio and assumed to have a constant value in this paper; $\kappa$ is the slope of swelling line in the $v-\ln p$ compression plane. The mean stress $p$, along with the deviatoric stress $q$, can be rigorously expressed as follows for the cylindrical cavity expansion problem (Chen and Abousleiman 2013)

$$
p=\frac{\sigma_{r}+\sigma_{\theta}+\sigma_{z}}{3}
$$




$$
q=\sqrt{\frac{1}{2}\left[\left(\sigma_{r}-\sigma_{\theta}\right)^{2}+\left(\sigma_{\theta}-\sigma_{z}\right)^{2}+\left(\sigma_{z}-\sigma_{r}\right)^{2}\right]}
$$

where $\sigma_{r}, \sigma_{\theta}$ and $\sigma_{z}$ denote the three principal stresses in the radial, tangential, and vertical directions, with compressive stresses being considered positive.

\section{Anisotropic modified Cam Clay model}

Based on the fundamental work equation and assumption of energy dissipation balance, Dafalias (1987) made a classical extension of the modified Cam Clay model (Roscoe and Burland 1968) from isotropy to anisotropy, through the introduction of a non-dimensional parameter $\alpha$ that measures the degree of plastic anisotropy of the soil in the triaxial space. The generalization of the model has also been proposed by Dafalias (1987) to include the threedimensional stress conditions, for which the scalar rotational hardening parameter $\alpha$ yet needs to be appropriately replaced by a second order deviatoric tensor $\alpha_{i j}$. Fig. 2 shows a schematic illustration of the anisotropic modified Cam Clay model in the principal stress space and in the triaxial $p-q$ plane as well.

As pointed out in Chen and Liu (2018), for a cylindrical cavity expanded in $K_{0}$ consolidated, initially transversely isotropic soils, all the three off-diagonal components of the deviatoric anisotropic tensor $\alpha_{i j}$, i.e., $\alpha_{r \theta}, \alpha_{r z}$, and $\alpha_{\theta z}$, must remain vanishing throughout the entire expansion process due to the axisymmetric nature of the problem. Dafalias' (1987) anisotropic Cam Clay model for such a particular boundary value problem, therefore, may be formulated as follows

$$
\begin{aligned}
F\left(\sigma_{r}, \sigma_{\theta}, \sigma_{z}, p_{c}, \alpha_{r}, \alpha_{\theta}, \alpha_{z}\right)=p^{2}-p \cdot p_{c}+\frac{3}{2 M^{2}}\left[\left(s_{r}-p \cdot \alpha_{r}\right)^{2}\right. \\
\left.+\left(s_{\theta}-p \cdot \alpha_{\theta}\right)^{2}+\left(s_{z}-p \cdot \alpha_{z}\right)^{2}+\left(p_{c}-p\right) p\left(\alpha_{r}^{2}+\alpha_{\theta}^{2}+\alpha_{z}^{2}\right)\right]=0
\end{aligned}
$$




$$
\begin{aligned}
& D p_{c}=\Lambda \frac{v}{\lambda-\kappa} \frac{\partial F}{\partial p} p_{c} \\
& D \alpha_{r}=\Lambda \cdot \frac{\partial F}{\partial \sigma_{k k}} \operatorname{sign}\left(\frac{\partial F}{\partial p}\right) \frac{v}{\lambda-\kappa} \frac{c}{p_{c}}\left(s_{r}-x p \alpha_{r}\right) \\
& D \alpha_{\theta}=\Lambda \cdot \frac{\partial F}{\partial \sigma_{k k}} \operatorname{sign}\left(\frac{\partial F}{\partial p}\right) \frac{v}{\lambda-\kappa} \frac{c}{p_{c}}\left(s_{\theta}-x p \alpha_{\theta}\right) \\
& D \alpha_{z}=\Lambda \cdot \frac{\partial F}{\partial \sigma_{k k}} \operatorname{sign}\left(\frac{\partial F}{\partial p}\right) \frac{v}{\lambda-\kappa} \frac{c}{p_{c}}\left(s_{z}-x p \alpha_{z}\right)
\end{aligned}
$$

where $F$ represents the yield function; $\lambda$ is the slope of normal compression line; $p_{c}$ is the volumetric hardening parameter denoting the preconsolidation pressure and essentially controlling the size of the distorted ellipsoidal yield surface, see the lower half of Fig. $2 ; M$ is the critical state ratio; $x$ and $c$ are two additional model constants pertinent to the level and pace of the plastic anisotropy development; $\Lambda$ is the plastic multiplier; sign stands for the signum function, $s_{r}=\sigma_{r}-p, s_{\theta}=\sigma_{\theta}-p$, and $s_{z}=\sigma_{z}-p$ give the radial, tangential, and vertical deviatoric stresses; $\alpha_{r}, \alpha_{\theta}$, and $\alpha_{z}$ correspond to the three non-zero components of the anisotropic tensor in the radial, tangential, and vertical directions, respectively; and $D \alpha_{r}, D \alpha_{\theta}$, and $D \alpha_{z}$ denote the associated infinitesimal changes of a given soil particle.

\section{Cavity expansion boundary value problem}

\section{GOVERNING EQUILIBRIUM EQUATION}

At each point in the deformed configuration, the stress equilibrium condition can be expressed as

$$
\frac{d \sigma_{r}}{d r}+\frac{\sigma_{r}-\sigma_{\theta}}{r}=0
$$

in which $r$ is the current radial position of a soil particle and $\frac{d \sigma_{r}}{d r}$ denotes the spatial derivative of $\sigma_{r}$ along the radial direction. Note that Eq. (9) holds for both the elastic and plastic regions (Fig. 
$1)$.

\section{SOLUTION IN ELASTIC REGION}

In the elastic region $r \geq r_{p}$, the displacements and strains are very small so an infinitesimal deformation will be assumed for the soil mass. For any material point currently located at $r$ in the elastic region, during the cavity expansion process, its radial and tangential strain increments $D \varepsilon_{r}$ and $D \varepsilon_{\theta}$ can be written as (compression taken as positive)

$$
\begin{aligned}
& D \varepsilon_{r}=-\frac{d\left(D u_{r}\right)}{d r} \\
& D \varepsilon_{\theta}=-\frac{D u_{r}}{r}
\end{aligned}
$$

where $D u_{r}$ denotes the differential displacement of the given material point in the radial direction, while $\frac{d}{d r}$ should be understood as the spatial derivative with respect to the radial coordinate $r$ (Eulerian description). These two incremental strains together with the one in $z$ direction, $D \varepsilon_{z}$, under the isotropic assumption of elastic behaviour, are related to the stress increments $D \sigma_{r}, D \sigma_{\theta}$, and $D \sigma_{z}$ of the particle as follows

$$
\left\{\begin{array}{l}
D \varepsilon_{r} \\
D \varepsilon_{\theta} \\
D \varepsilon_{z}
\end{array}\right\}=\frac{1}{E}\left[\begin{array}{ccc}
1 & -\mu & -\mu \\
-\mu & 1 & -\mu \\
-\mu & -\mu & 1
\end{array}\right] \cdot\left\{\begin{array}{l}
D \sigma_{r} \\
D \sigma_{\theta} \\
D \sigma_{z}
\end{array}\right\}
$$

where the Young's modulus $E$, as described earlier, shall be mean stress and specific volume dependent.

At the beginning of the expansion of cavity, the deformation of the soil is purely elastic with the Young's modulus $E$ and shear modulus $G$ equal to their initial values $E_{0}$ and $G_{0}$, respectively. Following the theory of elasticity (Timoshenko and Goodier 1970), the first instant incremental solutions for the stresses and radial displacement can be easily found to be 


$$
\begin{aligned}
& D \sigma_{r}=D \sigma_{a}\left(\frac{a_{0}}{r}\right)^{2} \\
& D \sigma_{\theta}=-D \sigma_{a}\left(\frac{a_{0}}{r}\right)^{2} \\
& D \sigma_{z}=0 \\
& D u_{r}=\frac{D \sigma_{a}}{2 G_{0}} \frac{a_{0}^{2}}{r}
\end{aligned}
$$

where the notation $D \sigma_{a}$ is used for the radial stress increment at the cavity wall over its initial value of $\sigma_{h}$. It should be emphasized that the above expressions are valid only in case that $E$ and $G$ are constants for any material particle in the elastic zone.

From Eqs. (13)-(15) one has

$$
D p=\frac{1}{3}\left(D \sigma_{r}+D \sigma_{\theta}+D \sigma_{z}\right)=0
$$

which combined with Eq. (12) gives

$$
D \varepsilon_{v}=D \varepsilon_{r}+D \varepsilon_{\theta}+D \varepsilon_{z}=\frac{3(1-2 \mu)}{E} D p=0
$$

where $D \varepsilon_{v}=-\frac{D v}{v}$ is the volumetric strain increment.

Eqs. (17) and (18) indicate that both the specific volume and mean stress must remain constants for any point in the elastic region when the internal cavity pressure is increased by an infinitesimal amount of $D \sigma_{a}$. This is a desirable feature and indeed a natural outcome of the tackled cylindrical cavity boundary value problem (Chen 2012). As a consequence, from Eq. (1) $E$ and $G$ should also stay constants after this loading increment. Eqs. (13)-(16) therefore can again be used to obtain the corresponding stress and displacement increments for the next increment of the cavity pressure, which invariably ends up with constant Young's modulus and shear modulus equal to $E_{0}$ and $G_{0}$, respectively, in the whole elastic region. The process can thus be repeated until the cavity pressure is finally increased to the current value of $\sigma_{a}$. The only point 
however to note is that, after the plastic zone has been formed outside the cavity, $a_{0}$ and $D \sigma_{a}$ appearing in Eqs. (13)-(16) should be replaced, respectively, by $r_{p x}$ pertaining to the instantaneous position of the particle which is originally located at $r_{p 0}$ while currently at the elastic-plastic interface $r_{p}$ and the corresponding radial stress increment at $r=r_{p x}$.

From the above analysis it is quite evident that the Young's modulus and shear modulus always remain unchanged in the external elastic region, throughout the cavity expansion. The solutions for stresses and radial displacement thus can be directly obtained as (Timoshenko and Goodier 1970)

$$
\begin{aligned}
& \sigma_{r}=\sigma_{h}+\left(\sigma_{p}-\sigma_{h}\right)\left(\frac{r_{p}}{r}\right)^{2} \\
& \sigma_{\theta}=\sigma_{h}-\left(\sigma_{p}-\sigma_{h}\right)\left(\frac{r_{p}}{r}\right)^{2} \\
& \sigma_{z}=\sigma_{v} \\
& u_{r}=\frac{\sigma_{p}-\sigma_{h}}{2 G_{0}} \frac{r_{p}^{2}}{r}
\end{aligned}
$$

where $\sigma_{p}$ denotes the radial stress at the elastic-plastic interface $r=r_{p}$.

\section{SOLUTION IN PLASTIC REGION}

\section{Elastoplastic constitutive relationship}

Consider now the plastic region as indicated in Fig. 1, which will progressively develop and spread from the cavity surface to the surrounding soils when the internal pressure $\sigma_{a}$ is gradually increased above $\sigma_{p}$. In this region, the total strain increments $D \varepsilon_{r}, D \varepsilon_{\theta}$, and $D \varepsilon_{z}$ may be written as the sum of the elastic and plastic components

$$
D \varepsilon_{r}=D \varepsilon_{r}^{e}+D \varepsilon_{r}^{p}, \quad D \varepsilon_{\theta}=D \varepsilon_{\theta}^{e}+D \varepsilon_{\theta}^{p}, \quad D \varepsilon_{z}=D \varepsilon_{z}^{e}+D \varepsilon_{z}^{p}
$$


where the incremental elastic strains $D \varepsilon_{r}^{e}, D \varepsilon_{\theta}^{e}$, and $D \varepsilon_{z}^{e}$ can be readily obtained from the isotropic Hooke's law by reference to Eq. (12), while the incremental plastic strains $D \varepsilon_{r}^{p}, D \varepsilon_{\theta}^{p}$, and $D \varepsilon_{z}^{p}$ should be obtained from the flow rule via the plastic potential function. For anisotropic Cam Clay model with an associated flow rule, from Eq. (4) the three incremental plastic strains can be expressed as

$$
\begin{aligned}
& D \varepsilon_{r}^{p}=\Lambda \frac{\partial F}{\partial \sigma_{r}}=y\left(m_{r} D \sigma_{r}+m_{\theta} D \sigma_{\theta}+m_{z} D \sigma_{z}\right) m_{r} \\
& D \varepsilon_{\theta}^{p}=\Lambda \frac{\partial F}{\partial \sigma_{\theta}}=y\left(m_{r} D \sigma_{r}+m_{\theta} D \sigma_{\theta}+m_{z} D \sigma_{z}\right) m_{\theta} \\
& D \varepsilon_{z}^{p}=\Lambda \frac{\partial F}{\partial \sigma_{z}}=y\left(m_{r} D \sigma_{r}+m_{\theta} D \sigma_{\theta}+m_{z} D \sigma_{z}\right) m_{z}
\end{aligned}
$$

where

$$
\begin{aligned}
& m_{r}=\frac{\partial F}{\partial \sigma_{r}}=\frac{1}{3} \frac{\partial F}{\partial p}+\frac{3}{M^{2}}\left(s_{r}-p \alpha_{r}\right) \\
& m_{\theta}=\frac{\partial F}{\partial \sigma_{\theta}}=\frac{1}{3} \frac{\partial F}{\partial p}+\frac{3}{M^{2}}\left(s_{\theta}-p \alpha_{\theta}\right) \\
& m_{z}=\frac{\partial F}{\partial \sigma_{z}}=\frac{1}{3} \frac{\partial F}{\partial p}+\frac{3}{M^{2}}\left(s_{z}-p \alpha_{z}\right) \\
& y=-1 /\left\{\left[-p+\frac{3 p\left(\alpha_{r}^{2}+\alpha_{\theta}^{2}+\alpha_{z}^{2}\right)}{2 M^{2}} \frac{v}{\lambda-\kappa}\right] p_{c} \frac{\partial F}{\partial p}+\frac{3 p}{M^{2}} \frac{v}{\lambda-\kappa} \frac{c}{p_{c}}\left[\left(-s_{r}+p_{c} \alpha_{r}\right)\left(s_{r}-x p \alpha_{r}\right)\right.\right. \\
& \left.\left.\quad+\left(-s_{\theta}+p_{c} \alpha_{\theta}\right)\left(s_{\theta}-x p \alpha_{\theta}\right)+\left(-s_{z}+p_{c} \alpha_{z}\right)\left(s_{z}-x p \alpha_{z}\right)\right]\left|\frac{\partial F}{\partial p}\right|\right\}
\end{aligned}
$$

and

$$
\frac{\partial F}{\partial p}=2 p-p_{c}+\frac{3}{2 M^{2}}\left\{-2\left(s_{r} \alpha_{r}+s_{\theta} \alpha_{\theta}+s_{z} \alpha_{z}\right)+p_{c}\left(\alpha_{r}^{2}+\alpha_{\theta}^{2}+\alpha_{z}^{2}\right)\right\}
$$

Eqs. (24a)-(24c) in combination with Eq. (12) hence lead to the desired elastoplastic constitutive equation as follows

$$
\left\{\begin{array}{l}
D \sigma_{r} \\
D \sigma_{\theta} \\
D \sigma_{z}
\end{array}\right\}=\frac{1}{\Delta}\left[\begin{array}{lll}
b_{11} & b_{12} & b_{13} \\
b_{21} & b_{22} & b_{23} \\
b_{31} & b_{32} & b_{33}
\end{array}\right]\left\{\begin{array}{l}
D \varepsilon_{r} \\
D \varepsilon_{\theta} \\
D \varepsilon_{z}
\end{array}\right\}
$$


where

$$
\begin{aligned}
b_{11} & =\frac{1}{E^{2}}\left[1-\mu^{2}+E m_{\theta}^{2} y+2 E \mu m_{\theta} m_{z} y+E m_{z}^{2} y\right] \\
b_{12}= & \frac{1}{E^{2}}\left[-E m_{r}\left(m_{\theta}+\mu m_{z}\right) y+\mu\left(1+\mu-E m_{\theta} m_{z} y+E m_{z}^{2} y\right)\right] \\
b_{13}= & \frac{1}{E^{2}}\left[-E m_{r}\left(\mu m_{\theta}+m_{z}\right) y+\mu\left(1+\mu+E m_{\theta}^{2} y-E m_{\theta} m_{z} y\right)\right] \\
b_{22}= & \frac{1}{E^{2}}\left[1-\mu^{2}+E m_{r}^{2} y+2 E \mu m_{r} m_{z} y+E m_{z}^{2} y\right] \\
b_{23}= & \frac{1}{E^{2}}\left[\mu+\mu^{2}+E \mu m_{r}^{2} y-E m_{\theta} m_{z} y-E \mu m_{r}\left(m_{\theta}+m_{z}\right) y\right] \\
b_{33}= & \frac{1}{E^{2}}\left[1-\mu^{2}+E m_{r}^{2} y+2 E \mu m_{r} m_{\theta} y+E m_{\theta}^{2} y\right] \\
b_{21}= & b_{12} \\
b_{31}= & b_{13} \\
b_{32}= & b_{23} \\
\Delta= & -\frac{1+\mu}{E^{3}}\left[\left(-1+\mu+2 \mu^{2}\right)+E(-1+\mu) m_{r}^{2} y+E(-1+\mu) m_{\theta}^{2} y-2 E \mu m_{\theta} m_{z} y\right. \\
& \left.-E m_{z}^{2} y+E \mu m_{z}^{2} y-2 E \mu m_{r}\left(m_{\theta}+m_{z}\right) y\right]
\end{aligned}
$$

which obviously are all explicit functions of the stress components $\sigma_{r}, \sigma_{\theta}$, and $\sigma_{z}$, specific volume $v$, volumetric hardening parameter $p_{c}$, and of the anisotropic hardening variables $\alpha_{r}, \alpha_{\theta}$, and $\alpha_{z}$. It is important for us to appreciate that the above equations (26) and (27a)-(27j) resemble closely the ones in Chen and Abousleiman (2013) for the Cam Clay constitutive model, with the only exception that $y, m_{r}, m_{\theta}$, and $m_{z}$ now should be replaced by Eqs. (25a)-(25e) pertinent to the present anisotropic model.

\section{Formulation within Eulerian framework}

One major contribution that was made by Chen and Abousleiman (2013) in solving the drained cavity expansion problem had been the introduction of an innovative, suitably chosen 
variable $\xi=\frac{u_{r}}{r}$, which enables converting the Eulerian equilibrium equation (9) to an equivalent Lagrangian form so as to be consistent with the already Lagangain-based constitutive relations (26). Following basically the similar procedure as proposed in Chen and Abousleiman (2013) and bearing in mind the resemblance of equilibrium and constitutive equations between the isotropic and anisotropic Cam Clay models, one may readily derive the following four governing differential equations for the three stresses and specific volume

$$
\begin{aligned}
& \frac{D \sigma_{r}}{D \xi}=-\frac{\sigma_{r}-\sigma_{\theta}}{1-\xi-\frac{v_{0}}{v(1-\xi)}} \\
& \frac{D \sigma_{\theta}}{D \xi}=-\frac{b_{21}}{b_{11}}\left[\frac{\sigma_{r}-\sigma_{\theta}}{1-\xi-\frac{v_{0}}{v(1-\xi)}}+\frac{b_{11}-b_{12}}{\Delta(1-\xi)}\right]-\frac{b_{22}-b_{21}}{\Delta(1-\xi)} \\
& \frac{D \sigma_{z}}{D \xi}=-\frac{b_{31}}{b_{11}}\left[\frac{\sigma_{r}-\sigma_{\theta}}{1-\xi-\frac{v_{0}}{v(1-\xi)}}+\frac{b_{11}-b_{12}}{\Delta(1-\xi)}\right]-\frac{b_{32}-b_{31}}{\Delta(1-\xi)} \\
& \frac{D v}{D \xi}=\frac{\Delta v}{b_{11}}\left[\frac{\sigma_{r}-\sigma_{\theta}}{1-\xi-\frac{v_{0}}{v(1-\xi)}}+\frac{b_{11}-b_{12}}{\Delta(1-\xi)}\right]
\end{aligned}
$$

where $\frac{D}{D \xi}$ is well known as the material derivative with respect to the auxiliary variable $\xi$, associated with the motion of a specific soil particle (Lagrangian description); and $v_{0}$ denotes the initial specific volume. It is observed that the right sides of Eqs. (28a)-(28d) all have been involved with and explicitly expressed as functions of the eight unknown variables, i.e., $\sigma_{r}, \sigma_{\theta}$, $\sigma_{z}, v, p_{c}, \alpha_{r}, \alpha_{\theta}$, and $\alpha_{z}$. Four additional differential equations that govern the three anisotropic hardening variables as well as the volumetric hardening variable therefore are required to make the problem fully solvable. This accomplishment will be described in the next subsection.

\section{Differential equations for evolution of plastic anisotropy}

The increment of plastic volumetric strain $D \varepsilon_{v}^{p}$ is simply given by

$$
D \varepsilon_{v}^{p}=D \varepsilon_{v}-D \varepsilon_{v}^{e}
$$


where $D \varepsilon_{v}^{e}$ denotes the elastic volumetric strain increment. Using the well-established relation (Schofield and Wroth 1968; Muir Wood 1990)

$$
D \varepsilon_{v}^{e}=\kappa \frac{D p}{v p}
$$

and the definition of $D \varepsilon_{v}=-\frac{D v}{v}$, Eq. (29) can be taken a further step to give

$$
D \varepsilon_{v}^{p}=\Lambda \frac{\partial F}{\partial p}=-\frac{D v}{v}-\kappa \frac{D p}{v p}
$$

Substituting Eq. (31) into Eqs. (5)-(8) and then combining with Eqs. (28a)-(28d), one may straightforwardly obtain the following expressions

$$
\begin{aligned}
& \frac{D p_{c}}{D \xi}=-\left\{\left(\frac{\Delta v}{b_{11}}-\frac{\kappa}{3 p}-\frac{\kappa b_{21}}{3 p b_{11}}-\frac{\kappa b_{31}}{3 p b_{11}}\right) \frac{\sigma_{r}-\sigma_{\theta}}{1-\xi-\frac{v_{0}}{v(1-\xi)}}\right. \\
& \left.+\left(\frac{\Delta v}{b_{11}}-\frac{\kappa b_{21}}{3 p b_{11}}-\frac{\kappa b_{31}}{3 p b_{11}}\right) \frac{b_{11}-b_{12}}{\Delta(1-\xi)}-\frac{\left(b_{22}-b_{21}\right)+\left(b_{32}-b_{31}\right)}{3 \Delta(1-\xi) p} \kappa\right\} \frac{p_{c}}{\lambda-\kappa} \\
& \frac{D \alpha_{r}}{D \xi}=-\left\{\left(\frac{\Delta v}{b_{11}}-\frac{\kappa}{3 p}-\frac{\kappa b_{21}}{3 p b_{11}}-\frac{\kappa b_{31}}{3 p b_{11}}\right) \frac{\sigma_{r}-\sigma_{\theta}}{1-\xi-\frac{v_{0}}{v(1-\xi)}}+\left(\frac{\Delta v}{b_{11}}-\frac{\kappa b_{21}}{3 p b_{11}}-\frac{\kappa b_{31}}{3 p b_{11}}\right) \frac{b_{11}-b_{12}}{\Delta(1-\xi)}\right. \\
& \left.-\frac{\left(b_{22}-b_{21}\right)+\left(b_{32}-b_{31}\right)}{3 \Delta(1-\xi) p} \kappa\right\} \operatorname{sign}\left[\frac{\partial F}{\partial p}\right] \frac{1}{\lambda-\kappa} \frac{c}{p_{c}}\left(s_{r}-x p \alpha_{r}\right) \\
& \frac{D \alpha_{\theta}}{D \xi}=-\left\{\left(\frac{\Delta v}{b_{11}}-\frac{\kappa}{3 p}-\frac{\kappa b_{21}}{3 p b_{11}}-\frac{\kappa b_{31}}{3 p b_{11}}\right) \frac{\sigma_{r}-\sigma_{\theta}}{1-\xi-\frac{v_{0}}{v(1-\xi)}}+\left(\frac{\Delta v}{b_{11}}-\frac{\kappa b_{21}}{3 p b_{11}}-\frac{\kappa b_{31}}{3 p b_{11}}\right) \frac{b_{11}-b_{12}}{\Delta(1-\xi)}\right. \\
& \left.-\frac{\left(b_{22}-b_{21}\right)+\left(b_{32}-b_{31}\right)}{3 \Delta(1-\xi) p} \kappa\right\} \operatorname{sign}\left[\frac{\partial F}{\partial p}\right] \frac{1}{\lambda-\kappa} \frac{c}{p_{c}}\left(s_{\theta}-x p \alpha_{\theta}\right) \\
& \frac{D \alpha_{z}}{D \xi}=-\left\{\left(\frac{\Delta v}{b_{11}}-\frac{\kappa}{3 p}-\frac{\kappa b_{21}}{3 p b_{11}}-\frac{\kappa b_{31}}{3 p b_{11}}\right) \frac{\sigma_{r}-\sigma_{\theta}}{1-\xi-\frac{v_{0}}{v(1-\xi)}}+\left(\frac{\Delta v}{b_{11}}-\frac{\kappa b_{21}}{3 p b_{11}}-\frac{\kappa b_{31}}{3 p b_{11}}\right) \frac{b_{11}-b_{12}}{\Delta(1-\xi)}\right. \\
& \left.-\frac{\left(b_{22}-b_{21}\right)+\left(b_{32}-b_{31}\right)}{3 \Delta(1-\xi) p} \kappa\right\} \operatorname{sign}\left[\frac{\partial F}{\partial p}\right] \frac{1}{\lambda-\kappa} \frac{c}{p_{c}}\left(s_{z}-x p \alpha_{z}\right)
\end{aligned}
$$

\section{INITIAL CONDITIONS}

Eqs. (28a)-(28d) and (32a)-(32d) present a system of simultaneous differential equations, with the numbers of unknown functions and equations both equal to eight. This set of equations 
can be solved as an initial value problem with the independent variable starting at $\xi=\xi_{p}$, provided that the associated initial values of $\sigma_{p}\left(\xi_{p}\right), \sigma_{\theta}\left(\xi_{p}\right), \sigma_{z}\left(\xi_{p}\right), v\left(\xi_{p}\right), p_{c}\left(\xi_{p}\right), \alpha_{r}\left(\xi_{p}\right)$, $\alpha_{\theta}\left(\xi_{p}\right)$, and $\alpha_{z}\left(\xi_{p}\right)$ are well specified. Here $\xi_{p}$ corresponds to the value of $\frac{u_{r}}{r}$ of a given particle, evaluated when it is just entering into the plastic state. It is easily seen that the initial conditions for the latter four hardening parameters are simply given by

$$
\begin{aligned}
& p_{c}\left(\xi_{p}\right)=p_{c, 0} \\
& \alpha_{r}\left(\xi_{p}\right)=\alpha_{r, 0} \\
& \alpha_{\theta}\left(\xi_{p}\right)=\alpha_{\theta, 0} \\
& \alpha_{z}\left(\xi_{p}\right)=\alpha_{z, 0}
\end{aligned}
$$

where $p_{c, 0}$ is the initial value of $p_{c}$ before the expansion of cavity; and $\alpha_{r, 0}, \alpha_{\theta, 0}$, and $\alpha_{z, 0}$ are, respectively, the components of the initial anisotropic hardening tensors in the radial, tangential, and vertical directions. While the required value of $\xi_{p}$ and the corresponding stress components as well as the specific volume can be fully determined from the already derived solutions for the outer elastic region, as is shown below.

First, it is evident that the stresses $\sigma_{r}\left(\xi_{p}\right), \sigma_{\theta}\left(\xi_{p}\right)$, and $\sigma_{z}\left(\xi_{p}\right)$ and the displacement $u_{r}\left(\xi_{p}\right)$ must satisfy the elastic region solutions given by Eqs. (19)-(22), which results in

$$
\begin{aligned}
& \sigma_{r}\left(\xi_{p}\right)+\sigma_{\theta}\left(\xi_{p}\right)=2 \sigma_{h} \\
& \sigma_{z}\left(\xi_{p}\right)=\sigma_{v} \\
& \xi_{p}=\frac{\sigma_{r}\left(\xi_{p}\right)-\sigma_{h}}{2 G_{0}} \\
& v\left(\xi_{p}\right)=v_{0}
\end{aligned}
$$

where Eq. (40) follows from Eq. (18).

On the other hand, at the instant that the material point just starts to yield the same stress 
components need to be located on the initial yield surface. Therefore, according to Eq. (4),

$$
\begin{aligned}
{\left[p\left(\xi_{p}\right)\right]^{2}-} & p\left(\xi_{p}\right) \cdot p_{c, 0}+\frac{3}{2 M^{2}}\left\{\left[s_{r}\left(\xi_{p}\right)-p\left(\xi_{p}\right) \cdot \alpha_{r, 0}\right]^{2}+\left[s_{\theta}\left(\xi_{p}\right)-p\left(\xi_{p}\right) \cdot \alpha_{\theta, 0}\right]^{2}\right. \\
& \left.+\left[s_{z}\left(\xi_{p}\right)-p\left(\xi_{p}\right) \cdot \alpha_{z, 0}\right]^{2}+\left[p_{c, 0}-p\left(\xi_{p}\right)\right] p\left(\xi_{p}\right)\left(\alpha_{r, 0}^{2}+\alpha_{\theta, 0}^{2}+\alpha_{z, 0}^{2}\right)\right\}=0
\end{aligned}
$$

where $p\left(\xi_{p}\right)=\frac{\sigma_{r}\left(\xi_{p}\right)+\sigma_{\theta}\left(\xi_{p}\right)+\sigma_{z}\left(\xi_{p}\right)}{3}$.

If Eqs. (37), (38), and (41) are combined it is found that

$$
\begin{aligned}
& \sigma_{r}\left(\xi_{p}\right)=\sigma_{h}+\sqrt{\left(\sigma_{h}-p_{0}\right)^{2}+\varpi} \\
& \sigma_{\theta}\left(\xi_{p}\right)=\sigma_{h}-\sqrt{\left(\sigma_{h}-p_{0}\right)^{2}+\varpi}
\end{aligned}
$$

where $\quad p_{0}=\frac{2 \sigma_{h}+\sigma_{v}}{3} \quad$ and $\quad \varpi=\frac{M^{2}}{3}\left(p_{0} p_{c, 0}-p_{0}^{2}\right)-2\left(p_{0}-\sigma_{h}\right)^{2}+2 p_{0} \alpha_{r, 0}\left(\sigma_{h}-p_{0}\right)-$ $\frac{1}{2}\left(\sigma_{z}^{\prime}-p_{0}^{\prime}\right)^{2}+p_{0}^{\prime} \alpha_{z, 0}\left(\sigma_{z}^{\prime}-p_{0}^{\prime}\right)-\frac{1}{2} p_{0}^{\prime} p_{c, 0}^{\prime}\left(\alpha_{r, 0}^{2}+\alpha_{\theta, 0}^{2}+\alpha_{z, 0}^{2}\right)$.

Eqs. (33)-(36), together with Eqs. (38)-(40) and (42)-(43), provide explicitly all the required initial conditions and suffice to solve the system of differential equations (28a)-(28d) and (32a)(32d) governing the anisotropic elastoplastic cavity expansion problem under drained conditions.

\section{SOLUTIONS IN CONNECTION WITH RADIAL COORDINATE}

At this stage it should be emphasized that the solutions resulting from Eqs. (28a)-(28d) and (32a)-(32d) are indeed expressed in relation to the auxiliary variable $\xi$ rather than to the particle position $r$. For completeness of the solution, a link between $\xi$ and $r$ is thus demanded and can be established following the conversion technique proposed by Chen and Abousleiman (2013) as

$$
\frac{r}{a}=e^{\int_{\xi(a)}^{\xi} \frac{d \xi}{1-\frac{v_{0}}{v(\xi)(1-\xi)}-\xi}}
$$

And for the particular position of the current elastic-plastic interface $r_{p}$, 


$$
\frac{r_{p}}{a}=e^{\int_{\xi(a)}^{\xi_{p}} \frac{d \xi}{1-\frac{v_{0}}{v(\xi)(1-\xi)}-\xi}}
$$

\section{Results and discussions}

With the aid of the symbolic computational software Mathematica 11.0, this section presents the numerical results for the cylindrical cavity expansion under drained condition in an anisotropic modified Cam Clay soil. Intensive parametric studies have been conducted firstly for verification of the proposed anisotropic formulations and solution scheme. Thereafter, the significant effects of $K_{0}$ consolidation as well as the subsequent stress-induced anisotropy are highlighted by making comparisons between Chen and Abousleiman's (2013) isotropic results and the current anisotropic ones. Finally, the influences of overconsolidation ratio $(O C R)$ on the stress patterns outside the cavity, the development of stress-induced anisotropy, as well as on the stress path followed by a soil particle are examined to some extent.

\section{VERIFICATION WITH EXISTING ISOTROPIC/ANISOTROPIC SOLUTIONS}

For the purposes of verification with the isotropic case (Chen and Abousleiman 2013), the current cavity expansion formulation/solution is implemented numerically in such a way that the controlling anisotropic plasticity parameters are taken sufficiently small, i.e., $c \rightarrow 0, \alpha_{r} \rightarrow 0$, $\alpha_{\theta} \rightarrow 0$, and $\alpha_{z} \rightarrow 0$. The reason for so doing instead of setting an exact zero value for these parameters is obvious, to retain the plastic anisotropy feature of the soil (though negligible) throughout the calculation and thus provide a true validation of the current anisotropic elastoplastic solution. On the other hand, to make comparison with the existing anisotropic

results for $K_{0}$ consolidated soils ( $\mathrm{Li}$ et al. 2017), the following values of $\alpha_{r, 0}=\alpha_{\theta, 0}=-\frac{1-K_{0}}{1+2 K_{0}}=$ 
$-0.17, \alpha_{z, 0}=\frac{2\left(K_{0}-1\right)}{1+2 K_{0}}=0.34$ and $c \rightarrow 0$ are specifically assigned. The parameters used for the calculation of this subsection are summarized in Table 1.

Fig. 3 compares the cavity expansion curve plotted in term of $\sigma_{a}$ versus $\frac{a}{a_{0}}$ as well as the propagation of elastic-plastic interface $\frac{r_{p}}{a}$ with respect to the normalized cavity radius $\frac{a}{a_{0}}$, between the current study and Chen and Abousleiman's (2013) isotropic solutions. Further comparisons for the distributions of three stress components $\sigma_{r}, \sigma_{\theta}, \sigma_{z}$ and specific volume $v$ along the radial distance $\frac{r}{a}$ (in relevance to an expanded radius of $\frac{a}{a_{0}}=2$ ) are provided in Fig. 4 for the isotropic case and Fig. 5 for the anisotropic case, respectively. From the overall excellent agreement between the current anisotropic solutions (full lines) and the published isotropic/anisotropic results (dots) by Chen and Abousleiman (2013) and Li et al. (2017), the validation of the present anisotropic formulations and solutions is evidently justified.

\section{INFLUENCES OF $\mathrm{K}_{0}$ CONSOLIDATION AND SUBSEQUENT STRESS-INDUCED ANISOTROPY}

The effects of initial $K_{0}$ consolidation and subsequent stress-induced anisotropy are evaluated on the cavity expansion curve (i.e., $\sigma_{a}-a$ relationship), distributions of stress components and specific volume, variations of the anisotropic and volumetric hardening parameters, and on the $p-q$ stress path for a soil element at the cavity wall as well. The soil parameters used in the calculations are those related to case $A$ with $O C R=1$, as described in Table 2.

Fig. 6 displays the calculated relationship between the cavity pressure $\sigma_{a}$ and the normalized cavity radius $\frac{a}{a_{0}}$. Also included in this figure are the counterpart isotropic results from Chen and Abousleiman (2013) in which the soil plastic anisotropy has been entirely neglected. It 
is obviously seen that, the use of isotropic modified Cam Clay model results in larger value of $\sigma_{a}$ required to expand the cavity than in the anisotropic case, and hence an overprediction of the limiting cavity pressure.

Fig. 7 shows the curves of $\sigma_{r}, \sigma_{\theta}, \sigma_{z}$, and $v$ against the normalized radial distance $\frac{r}{a}$ corresponding to an expanded cavity radius of $\frac{a}{a_{0}}=2$, and their comparisons with the existing isotropic solutions (Chen and Abousleiman 2013) as well. The figure reveals that ignoring the anisotropic behaviour of the soil, although having little impact on the general trends of the stress and volumetric variables, may result in appreciable overestimates or underestimates of these variables at some places. A comparison with the undrained cavity expansion (Chen and Liu 2018) however indicates that such effects of anisotropy are less significant for the present drained situation, especially if the magnitude of $\sigma_{r}$ is concerned.

Fig. 8 illustrates the distributions of hardening parameter $p_{c}$ and anisotropic variables $\alpha_{r}$, $\alpha_{\theta}, \alpha_{z}$ again for $\frac{a}{a_{0}}=2$, which actually also represent the evolving anisotropic plasticity of a soil particle due to the self-similarity nature of the cavity expansion problem. The continuing development of soil anisotropy is shown by the fact that $\alpha_{r}$ and $\alpha_{\theta}$ deviate gradually from each other with the accumulated plastic straining. In contrast, the isotropic assumption in Chen and Abousleiman (2013) has rendered the vanishing of anisotropy everywhere, i.e., $\alpha_{r}=\alpha_{\theta}=\alpha_{z} \equiv$ 0.

The stress paths in the $p-q$ plane followed by a soil element at the cavity surface are further plotted and compared in Fig. 9. For both anisotropic and isotropic Cam Clay models, the in situ stress state is represented by the same point $A$ with $p_{0}=69.9 \mathrm{kPa}$ and $q_{0}=44.9 \mathrm{kPa}$. During the cavity expansion, each of the two stress paths approaches asymptotically the critical state line from the "wet" side, with the anisotropic one targeting at a relatively lower stress level 
yet seemingly a faster pace. The current yield surfaces, meanwhile, become progressively larger so as to accommodate the plastic straining and new stress state, as indicated by the two yield loci passing through points $F$ and $F^{\prime}$ (Fig. 9). It is as expected that with the use of isotropic Cam Clay model, the yield locus always remains symmetric about the $p$ axis during the course of cavity expansion. However, the same would not be true for the anisotropic case. From Fig. 9, it is clearly seen that the initial solid yield locus passing $A$ is already inclined to the $p$ axis as a result of the $K_{0}$ consolidation. The magnitude of such inclination, on the other hand, must further change in accordance with the cavity expansion process to appropriately reflect the rotational hardening of the anisotropic elastoplastic model employed.

\section{INFLUENCES OF OVERCONSOLIDATION RATIO}

To facilitate the parametric analysis, three different values of $O C R=1,2$, and 4 are examined to investigate how it influences the responses of cavity. Here $O C R$ is defined as the ratio of past maximum effective vertical stress $\sigma_{v \text {,max }}$ to the in situ vertical effective stress $\sigma_{v}$, which is different from the isotropic overconsolidatoin ratio $R$ as used in Chen and Abousleiman (2013) in terms of the mean effective stress. The soil parameters for these three cases are given in Table 2, which have the same set-up as used in Chen and Liu (2018). Fig. 10 illustrates that the predicted magnitude of the cavity pressure $\sigma_{a}$ decreases with the increase of $O C R$, and so does the ultimate cavity pressure. Nevertheless, for all the three values of $O C R$ considered, the respective limiting cavity pressures are approached at almost the same expanded cavity size of $\frac{a}{a_{0}}=7$ or beyond.

Fig. 11 shows the variations of the stress components $\sigma_{r}, \sigma_{\theta}, \sigma_{z}$ and specific volume $v$ with the normalized radial distance $\frac{r}{a}$ for the three values of $O C R$ studied, all corresponding to $\frac{a}{a_{0}}=2$. 
The general trend in this figure is that the stresses increase with decreasing $\frac{r}{a}$ in the plastic region, except for the normally consolidated case of $O C R=1$ in which both $\sigma_{\theta}$ and $\sigma_{z}$ first tend to drop down to certain minimum values, and then climb up progressively in the closer vicinity of the cavity. It is found that all the three stress components at the cavity surface decreases monotonically with the increase of $O C R$, though probably not very markedly. For example, $\sigma_{r}(a)$ reduces from $254.0 \mathrm{kPa}$ to $226.7 \mathrm{kPa}$ as $O C R$ ranges from 1 to 4 , indicating that a lower cavity expansion pressure is required to double the cavity radius $\left(\frac{a}{a_{0}}=2\right)$ for a moderately overconsolidated soil. The observed somewhat steep slopes in the near-cavity parts of the $\sigma_{r}$ and $\sigma_{z}$ curves imply that the soil particles even very close to the cavity wall still have not reached the critical failure state, a similar phenomenon having been reported in Chen and Abousleiman (2013) for the solution of cavity expansion in isotropic Cam Clay soil. Finally, it is interesting to note that throughout the range of $O C R$ from 1 to 4 , the change in the specific volume $v$ is fairly modest and the soil undergoes merely compression during the whole process of expansion.

The impacts of $O C R$ on the distributions (or evolutions) of the hardening parameter $p_{c}$ and anisotropy variables $\alpha_{r}, \alpha_{\theta}, \alpha_{z}$ around the cavity, at the instant of $\frac{a}{a_{0}}=2$, are further given in Fig. 12. Note that due to the constraint condition $\alpha_{r}+\alpha_{\theta}+\alpha_{z}=0$ imposed to the anisotropy deviator tensor $\alpha_{i j}$, among the three rotational hardening variables as presented in the figure only two of them are in fact independent, i.e., the increase in $\alpha_{r}$ in some way must compensate for the reduction in $\alpha_{\theta}$ and $\alpha_{z}$ (Chen and Liu 2018). Also shown in Fig. 12 is the strictly monotonic increase in $p_{c}$ with the decrease of radial distance, for all the three values of $O C R$ considered, which therefore provides a clear indication of the general strain hardening behaviour of the surrounding soils that occurs in the course of cylindrical cavity expansion. 
Fig. 13 depicts the stress paths followed by a soil element at the cavity wall in the $p-q$ plane and in the deviatoric plane $\pi$ for $O C R=1,2$, and 4 . As would be expected, the in situ stress points are located on and inside the initial yield surfaces, respectively, for $O C R$ equal to and greater than 1 , see point $A$ and associated yield loci projected onto the $p-q$ and $\pi$ planes. In this figure $Y S_{I}$ and $Y S_{F}$ correspond to the sections perpendicular to the space diagonal, of the initial yield surface encompassing through point $A$ and of the final yield surface through the ending stress point $F$ when the expanded cavity radius $\frac{a}{a_{0}}=2$. Both the two cross sections can be proved to have a circular shape (Figs. 13d, 13e, and 13f) except for the specific case of $O C R=$ 1, for which the in situ stress point locates at the rightmost of the initial yield ellipsoid and therefore the projection of the ellipsoid simply shrinks to a point $(A)$ on the $\pi$ plane. As before, the $p-q$ stress path for $O C R=1$ (Fig. 13a) first moves upper-left and then turns upper-right towards the critical state line as cavity expansion proceeds, which is accompanied by a significant leftward move of the stress path in the deviatoric plane (Fig. 13d). The shapes of the stress curves observed for the overconsolidated cases of $O C R=2$ and 4 are similar to that for the normally consolidated soil $(O C R=1)$. The main distinct difference is that the $p-q$ stress paths (Figs. 13b and 13c) now must rise at constant $p$ until the initial yield loci are reached (at $C$ ) and the corresponding stress paths in the $\pi$ plane (Figs. 13e and 13f) go straight to the left due to the elastic deformation. The comparisons in Fig. 13 also show that as $O C R$ increases, the stress path tends toward the critical state line at relatively lower mean stress $p$ and deviatoric stress $q$. Note that in Fig. 13c the ellipse passing through point $C$, i.e., the cross section of the initial yield surface of ellipsoid with the plane containing both hydrostatic axis and yielding point $C$, is symmetric with respect to the $p$ axis. This, nevertheless, by no means indicates that the initial ellipsoidal yield surface is aligned with the hydrostatic axis as well. This point probably can be 
demonstrated much more clearly through Fig. $13 \mathrm{f}$ where the section of the yield surface $Y S_{I}$ is found indeed not centred at the origin on the $\pi$ plane. As a matter of fact, for all the three $O C R$ cases considered in the analysis, the initial yield surfaces are virtually identical, each of them having the same level of inclination/distortion in the stress space as a result of $K_{0}$ consolidation.

\section{Conclusions}

A semi-analytical elastoplastic solution for the drained expansion of a cylindrical cavity in $K_{0}$ consolidated soils is rigorously developed, based on Dafalias' representative anisotropic Cam Clay model that is capable of capturing both the inherent and stress-induced anisotropies. Following the novel solution scheme proposed earlier by Chen and Abousleiman (2013), an additional auxiliary variable has been introduced to establish a link between the Eulerian and Lagrangian formulations of the radial equilibrium condition. The plastic zone solution can be eventually obtained by solving numerically a system of eight partial differential equations, which essentially involves the three stress components and three anisotropic hardening parameters both in radial, tangential, and vertical directions, specific volume, as well as the preconsolidation pressure.

The present anisotropic formulations and solutions are verified against the existing isotropic results in terms of the overall cavity responses and of the distributions of stress components and specific volume. Numerical results demonstrate that neglecting the anisotropic behaviour of the soil may lead to appreciable overestimates or underestimates of these stress and volumetric variables at some places. The great impacts of overconsolidation ratio are also clearly observed on the stress patterns outside the cavity, the development of stress-induced anisotropy, and on the stress paths as well. It is shown that the stress path tends toward the critical state line at relatively lower mean and deviatoric stresses as $O C R$ increases. The proposed analytical solution provides 
a useful benchmark from which the numerical results may be evaluated for various geotechnical boundary value problems involving the sophisticated anisotropic critical state plasticity models.

\section{Acknowledgements}

This research is funded by Louisiana Transportation Research Center (Grant No. DOTLT1000208) and Transportation Innovation for Research Exploration Program [TIRE], Louisiana Transportation Research Center (Grant No. DOTLT1000135).

\section{References}

Banerjee, P.K., and Yousif, N.B. 1986. A plasticity model for the mechanical behaviour of anisotropically consolidated clay. Int. J. Numer. Anal. Meth. Geomech., 10(5): 521-541.

Carter, J.P., Booker, J.R., and Yeung, S.K. 1986. Cavity expansion in cohesive frictional soils. Geotechnique, 36(3): 349-358.

Chen, S.L. 2012. Analytical and numerical analysis of wellbore drilled in elastoplastic porous formations. Ph.D. thesis, The University of Oklahoma, Norman, OK, USA.

Chen, S.L., and Abousleiman, Y.N. 2012. Exact undrained elasto-plastic solution for cylindrical cavity expansion in modified Cam Clay soil. Geotechnique, 62(5): 447-456.

Chen, S.L., and Abousleiman, Y.N. 2013. Exact drained solution for cylindrical cavity expansion in modified Cam Clay soil. Geotechnique, 63(6): 510-517.

Chen, S.L., and Abousleiman, Y.N. 2016. Drained and undrained analyses of cylindrical cavity contractions by bounding surface plasticity. Can. Geotech. J., 53(9): 1398-1411.

Chen, S.L., and Abousleiman, Y.N. 2018. Cavity expansion in strain hardening frictional soils under drained condition. Int. J. Numer. Anal. Meth. Geomech., 42(1): 132-142.

Chen, S.L., and Liu, K. 2018. Undrained cylindrical cavity expansion in anisotropic critical state 
soils. Geotechnique, ahead of print. doi: 10.1680/jgeot.16.p.335.

Collins, I.F., and Yu, H.S. 1996. Undrained cavity expansion in critical state soils. Int. J. Numer. Anal. Mech. Geomech., 20(7): 489-516.

Cudmani, R., and Osinov, V.A. 2001. The cavity expansion problem for the interpretation of cone penetration and pressuremeter tests. Can. Geotech. J., 38(3): 622-638.

Dafalias, Y.F. 1987. An anisotropic critical state clay plasticity model. Proceedings of the Constitutive Laws for Engineering Materials: Theory and Applications, Tucson, AZ, USA (eds C.S. Desai, E. Krempl, P.D. Kiousis and T. Kundu), pp. 513-521. New York, USA: Elsevier.

Gibson, R.E., and Anderson, W.F. 1961. In situ measurement of soil properties with the pressuremeter. Civ. Engng. Publ. Wks. Rev., 56(658): 615-618.

Kolymbas, D., Wagner, P., and Blioumi, A. 2012. Cavity expansion in cross-anisotropic rock. Int. J. Numer. Anal. Meth. Geomech, 36(2): 128-139.

Ladanyi, B., and Foriero, A. 1998. A numerical solution of cavity expansion problem in sand based directly on experimental stress-strain curves. Can. Geotech. J., 35(4): 541-559.

Li, J.P., Gong, W.B., Li, L., and Liu F. (2017). Drained elastoplastic solution for cylindrical cavity expansion in K0-consolidated anisotropic soil. J. Eng. Mech., 143(11): 04017133, 1-10.

Li, L., Li, J.P., and Sun, D.A. 2016. Anisotropically elasto-plastic solution to undrained cylindrical cavity expansion in K0-consolidated clay. Comput. Geotech., 73: 83-90.

Mo, P.Q., and Yu, H.S. 2017a. Drained cavity expansion analysis with a unified state parameter model for clay and sand. Can. Geotech. J. doi: 10.1139/cgj-2016-0695.

Mo, P.Q., and Yu, H.S. 2017b. Undrained cavity contraction analysis for prediction of soil behavior around tunnels. Int. J. Geomech., 17(5): 04016121. 
Muir Wood, D.M. 1990. Soil behaviour and critical state soil mechanics. Cambridge University Press, Cambridge, UK.

Roscoe, K.H., and Burland, J.B. 1968. On the generalized stress-strain behaviour of wet clays. Engineering Plasticity, edited by J. Heyman and F.A. Leckie, 535-608, Cambridge. University Press.

Schofield, A.N., and Wroth, C.P. 1968. Critical state soil mechanics. McGraw-Hill, London, UK.

Sivasithamparam, N., and Castro, J. 2018. Undrained expansion of a cylindrical cavity in clays with fabric anisotropy: theoretical solution [online]. Acta Geotech. doi: https://doi.org/10.1007/ s11440-017-0587-4

Tavernas, F., and Leroueil, S. 1977. The effect of stresses and time on yielding of clays. Proc. 9th Int Conf Soil Mechanics and Foundation Engineering, Tokyo, 1, 319-326.

Timoshenko, S.P., and Goodier, J.N. 1970. Theory of elasticity. McGraw-Hill, New York, N.Y.

Vesic, A.C. 1972. Expansion of cavities in infinite soil mass. J. Soil Mech. Found. Div.-ASCE, 98(SM3): 265-290.

Voyiadjis, G.Z., and Song, C.R. 2000. Finite strain, anisotropic modified Cam clay model with plastic spin I: theory. J. Eng. Mech.-ASCE, 126(10): 1012-1019.

Wheeler, S.J., Näätänen, A., Karstunen, M., and Lojander, M. 2003. An anisotropic elastoplastic model for soft clays. Can. Geotech. J., 40(2): 403-418.

Yu, H.S. 2000. Cavity expansion methods in geomechanics. Kluwer Academic Publisher, Dordrecht, the Netherlands. 


\section{Captions of tables and figures}

Table 1. Parameters used for verification with isotropic solution

Table 2. Parameters used in numerical analyses for anisotropic case

Fig. 1. Expansion of cavity in anisotropic Cam Clay soil

Fig. 2. Yield surface in (a) principal stress space (fixed directions); (b) triaxial p-q plane for anisotropic model

Fig. 3. Comparisons between isotropic and anisotropic solutions: variations of cavity pressure $\sigma_{a}$ and elastic-plastic interface $r_{p}$ with normalized cavity radius, OCR $=1.06$

Fig. 4. Comparisons between isotropic and anisotropic solutions: variations of the three stress components $\sigma_{r}, \sigma_{\theta}, \sigma_{z}$ and specific volume $v$ with radial distance, $\mathrm{OCR}=1.06$

Fig. 5. Comparisons of the stress components $\sigma_{r}, \sigma_{\theta}, \sigma_{z}$ and specific volume $v$ for anisotropic case $(O C R=1.06)$

Fig. 6. Influences of $K_{0}$ consolidation anisotropy on the cavity expansion curve $\left(\sigma_{a}-a\right.$ relationship)

Fig. 7. Influences of $K_{0}$ consolidation anisotropy on the distributions of $\sigma_{r}, \sigma_{\theta}, \sigma_{z}$ and $v$ around the cavity [solid and dashed lines represent anisotropic and isotropic (Chen and Abousleiman, 2013) solutions, respectively]

Fig. 8. Influences of $K_{0}$ consolidation anisotropy on the distributions of $\alpha_{r}, \alpha_{\theta}, \alpha_{z}$ and $p_{c}$ around the cavity [solid and dashed lines represent anisotropic and isotropic solutions, respectively]

Fig. 9. Influences of $K_{0}$ consolidation anisotropy on the p-q stress path

Fig. 10. Influences of overconsolidation ratio on the cavity expansion curve 
Fig. 11. Distributions of radial, tangential, vertical stresses and specific volume along the radial distance: (a) $\mathrm{OCR}=1$; (b) $\mathrm{OCR}=2$; and (c) $\mathrm{OCR}=4$

Fig. 12. Distributions of hardening parameter $p_{c}$ and anisotropic variables $\alpha_{r}, \alpha_{\theta}, \alpha_{z}$ along the radial distance: (a) $\mathrm{OCR}=1$; (b) $\mathrm{OCR}=2$; and (c) $\mathrm{OCR}=4$

Fig. 13. Stress path followed by a soil particle at cavity wall in p-q plane and deviatoric plane: (a) and (d) OCR =1; (b) and (e) OCR = 2; (c) and (f) OCR $=4$ 
Table 1. Parameters used for verification with isotropic solution

\begin{tabular}{cccccccc}
\hline \multicolumn{7}{c}{$M=1.2, \lambda=0.15, \kappa=0.03, v=0.278$, and $v_{c s}=2.74$} \\
\hline OCR & $\sigma_{h}: \mathrm{kPa}$ & $\sigma_{v}: \mathrm{kPa}$ & $p_{0}: \mathrm{kPa}$ & $q_{0}: \mathrm{kPa}$ & $K_{0}$ & $v_{0}$ & $G_{0}: \mathrm{kPa}$ \\
\hline 1.06 & 100 & 160 & 120 & 60 & 0.625 & 2.06 & 4302 \\
\hline
\end{tabular}


Table 2. Parameters used in numerical analyses for anisotropic case

\begin{tabular}{ccccccccccc}
\hline \multicolumn{1}{c}{$M=1.2, \lambda=0.15, \kappa=0.03, v_{c s}=2.74, c=2, x=2.3, \alpha_{r, 0}=\alpha_{\theta, 0}=-0.21, \alpha_{z, 0}=0.42$} \\
\hline Case & OCR & $v_{0}$ & $K_{0, n c}$ & $K_{0,0 c}$ & $\begin{array}{c}G_{0} \\
(\mathrm{kPa})\end{array}$ & $\begin{array}{c}\sigma_{h} \\
(\mathrm{kPa})\end{array}$ & $\begin{array}{c}\sigma_{v} \\
(\mathrm{kPa})\end{array}$ & $\begin{array}{c}p_{0} \\
(\mathrm{kPa})\end{array}$ & $\begin{array}{c}q_{0} \\
(\mathrm{kPa})\end{array}$ \\
\hline $\mathrm{A}$ & 1 & 2.16 & 0.55 & 0.55 & 2516 & 54.91 & 99.84 & 69.89 & 44.93 \\
\hline $\mathrm{B}$ & 2 & 2.16 & 0.55 & 0.70 & 2822 & 39.19 & 55.99 & 44.79 & 16.80 \\
\hline $\mathrm{C}$ & 4 & 2.16 & 0.55 & 1.00 & 3094 & 30.94 & 30.94 & 30.94 & 0 \\
\hline
\end{tabular}




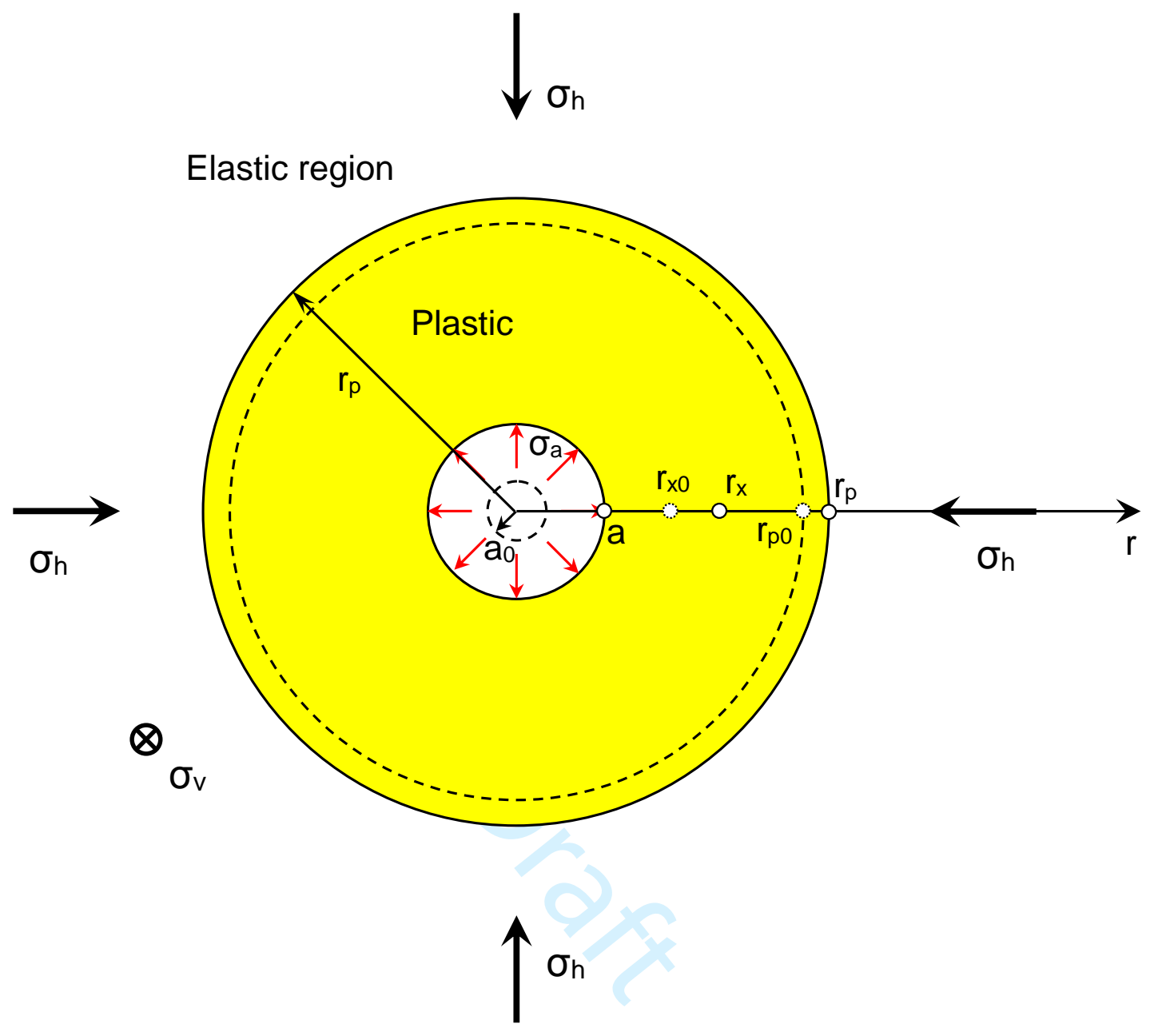

Fig. 1. Expansion of cavity in anisotropic Cam Clay soil 

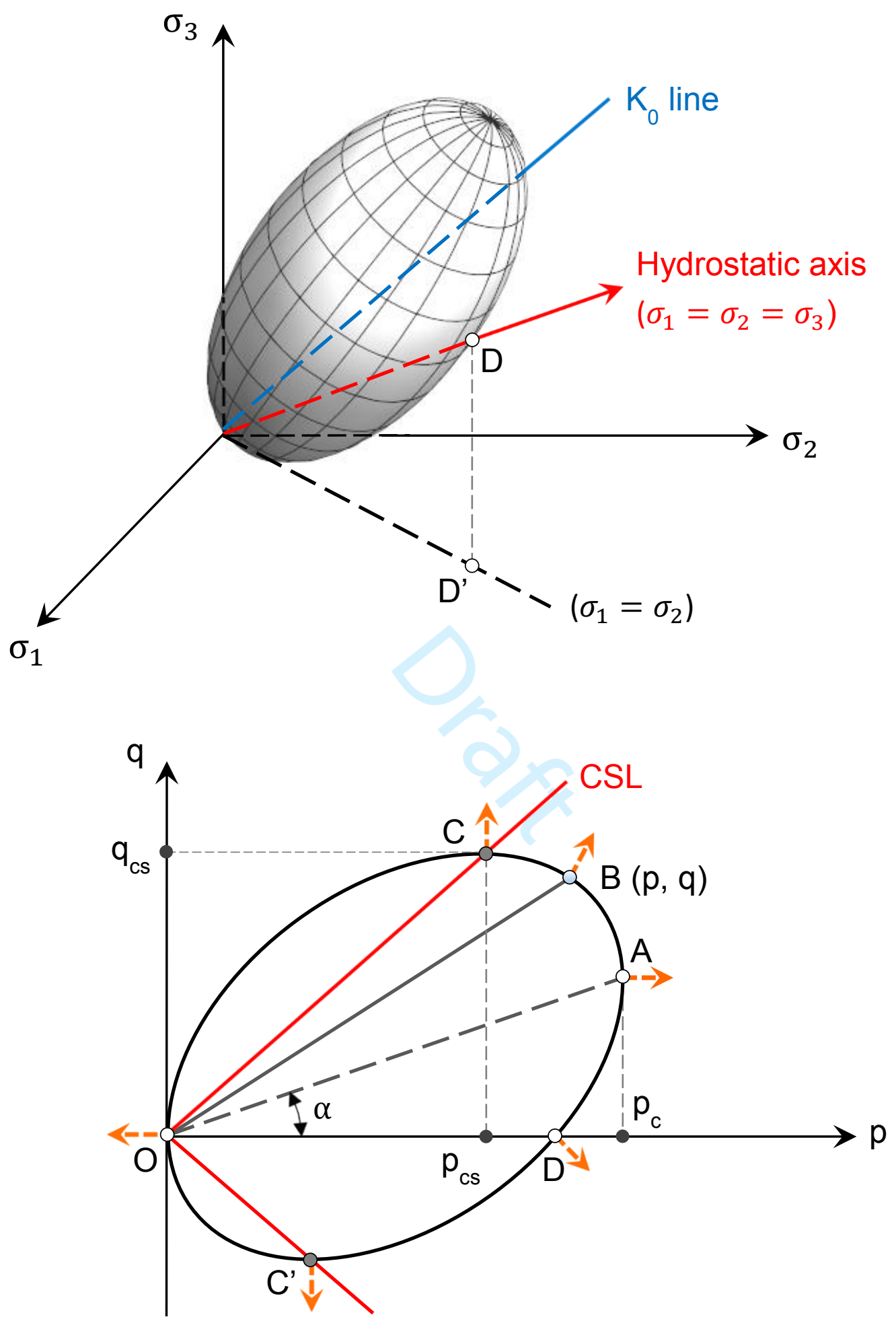

(b)

Fig. 2. Yield surface in (a) principal stress space (fixed directions); (b) triaxial p-q plane for anisotropic model 


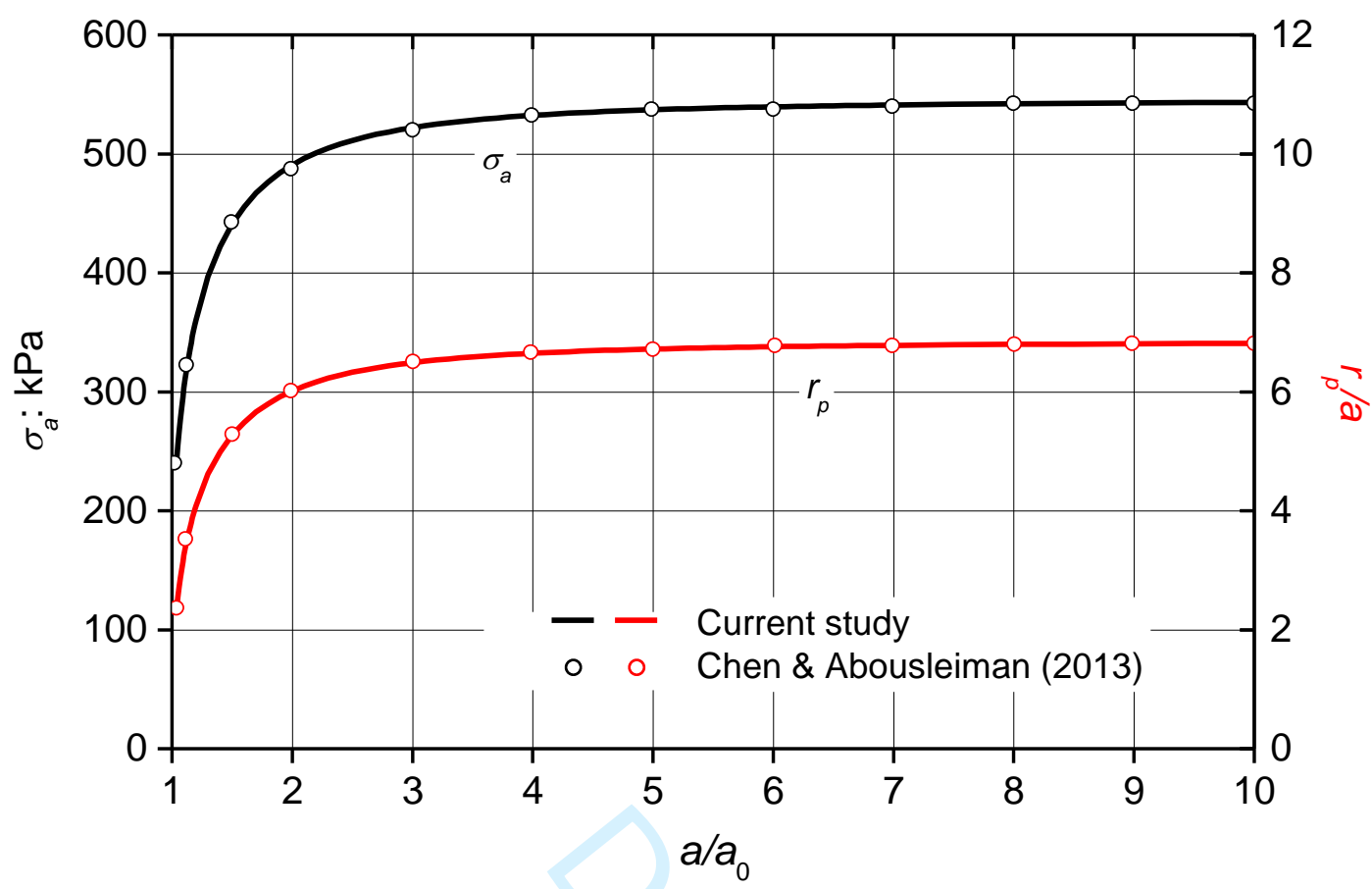

Fig. 3. Comparisons between isotropic and anisotropic solutions: variations of cavity pressure $\sigma_{a}$ and elastic-plastic interface $r_{p}$ with normalized cavity radius, OCR $=1.06$ 


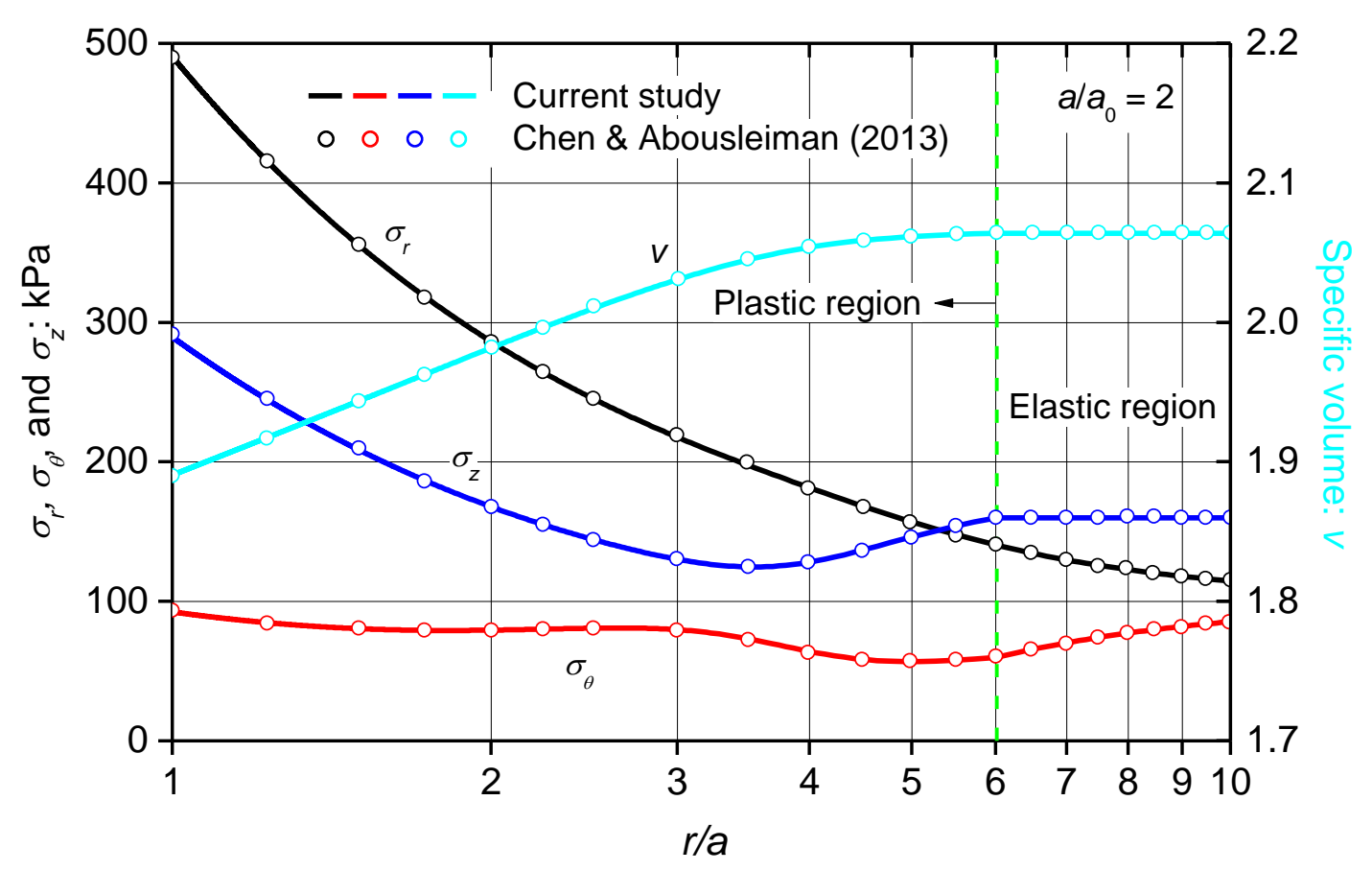

Fig. 4. Comparisons between isotropic and anisotropic solutions: variations of the three stress components $\sigma_{r}, \sigma_{\theta}, \sigma_{z}$ and specific volume $v$ with radial distance, $\mathrm{OCR}=1.06$ 


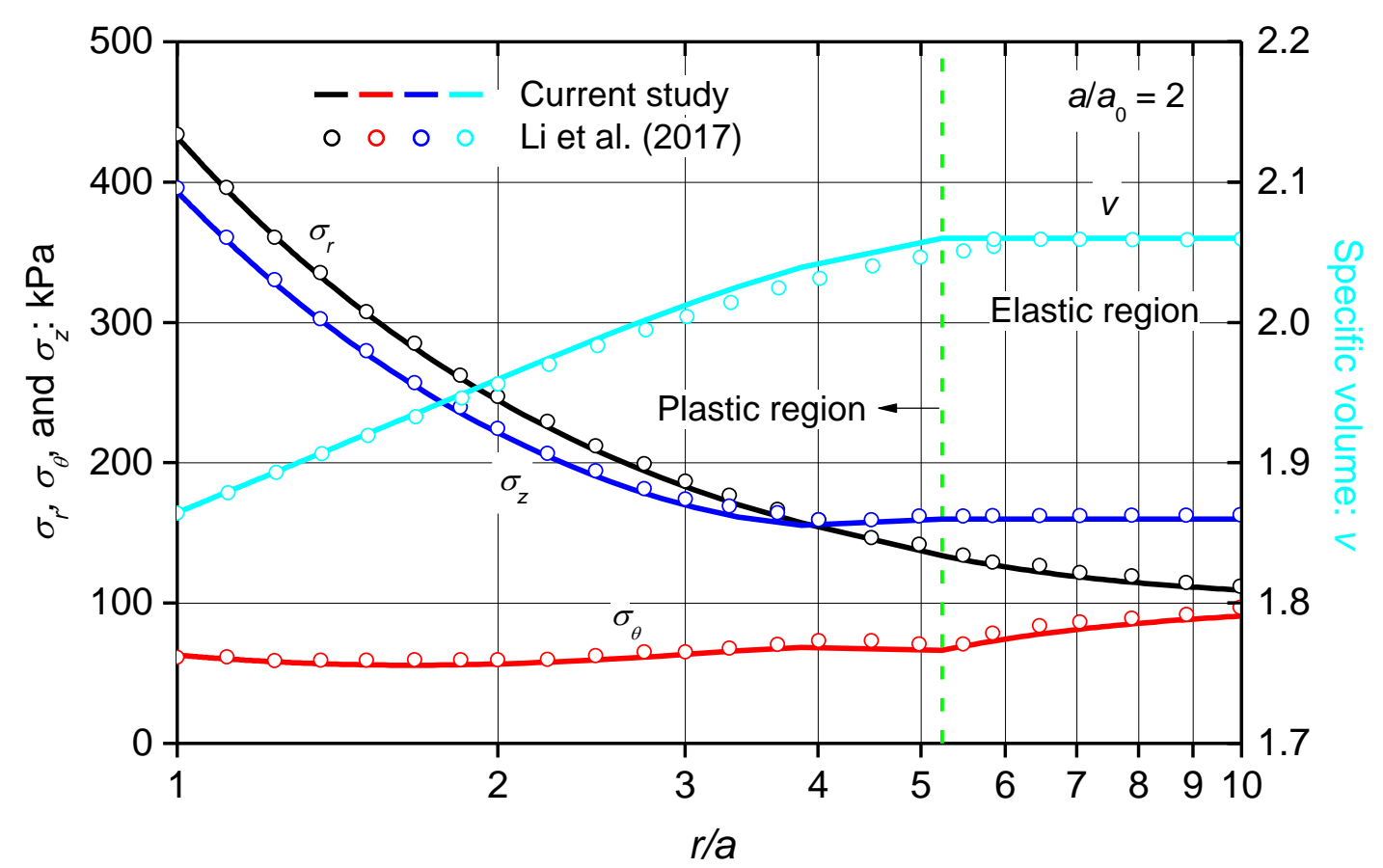

Fig. 5. Comparisons of the stress components $\sigma_{r}, \sigma_{\theta}, \sigma_{z}$ and specific volume $v$ for anisotropic case $(O C R=1.06)$ 


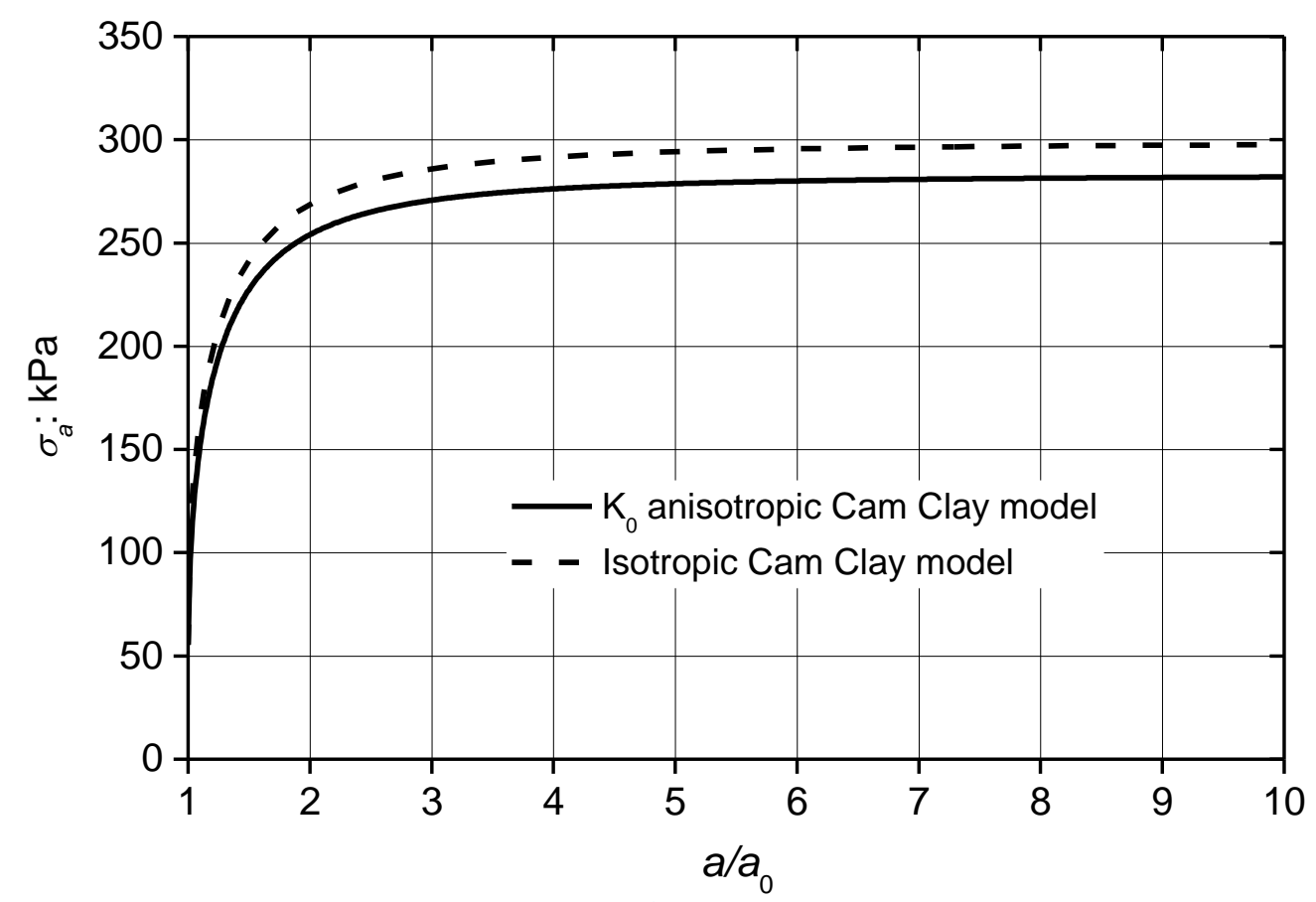

Fig. 6. Influences of $K_{0}$ consolidation anisotropy on the cavity expansion curve ( $\sigma_{a}-a$ relationship) 


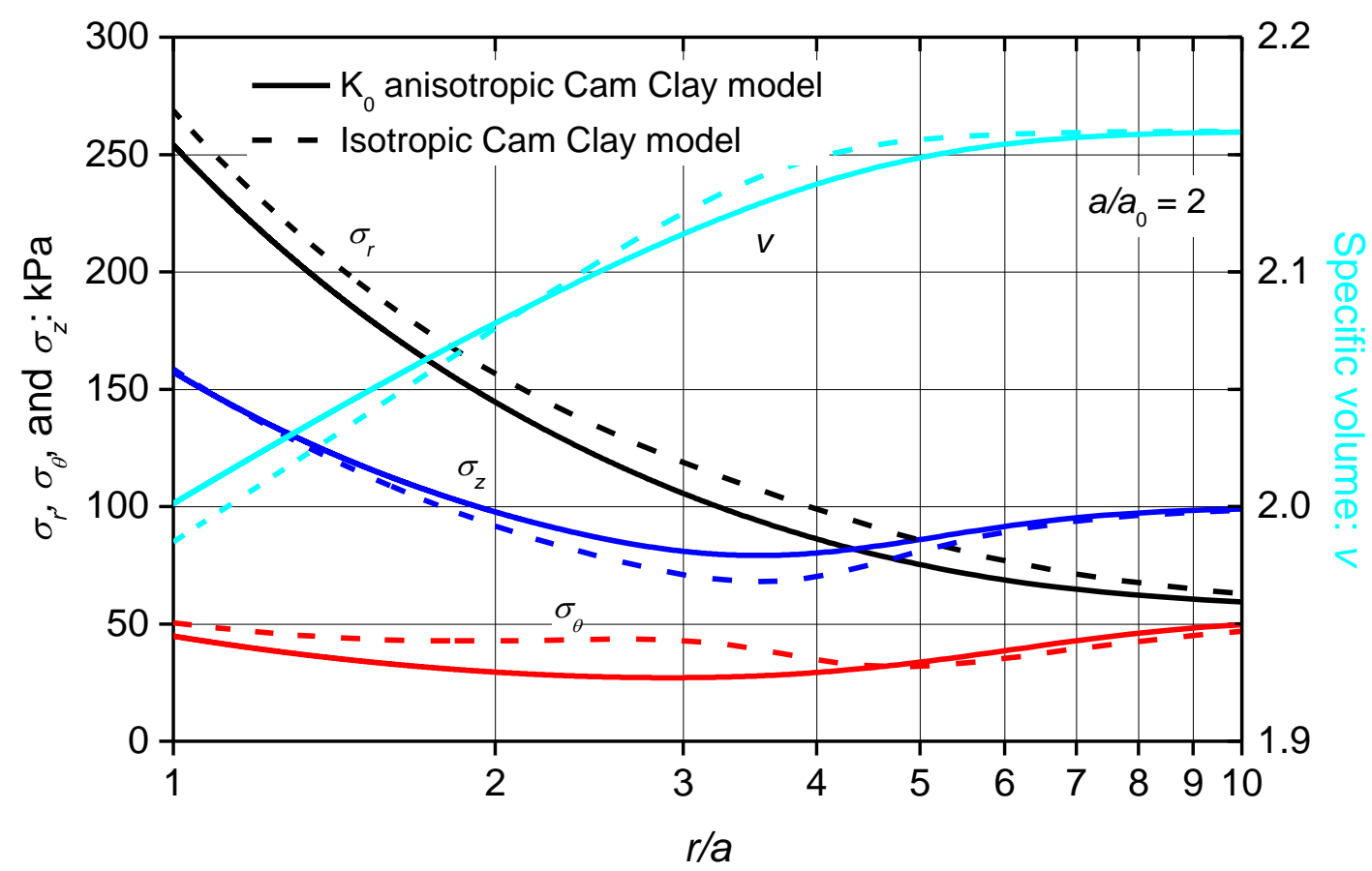

Fig. 7. Influences of $K_{0}$ consolidation anisotropy on the distributions of $\sigma_{r}, \sigma_{\theta}, \sigma_{z}$ and $v$ around the cavity [solid and dashed lines represent anisotropic and isotropic (Chen and Abousleiman, 2013) solutions, respectively] 


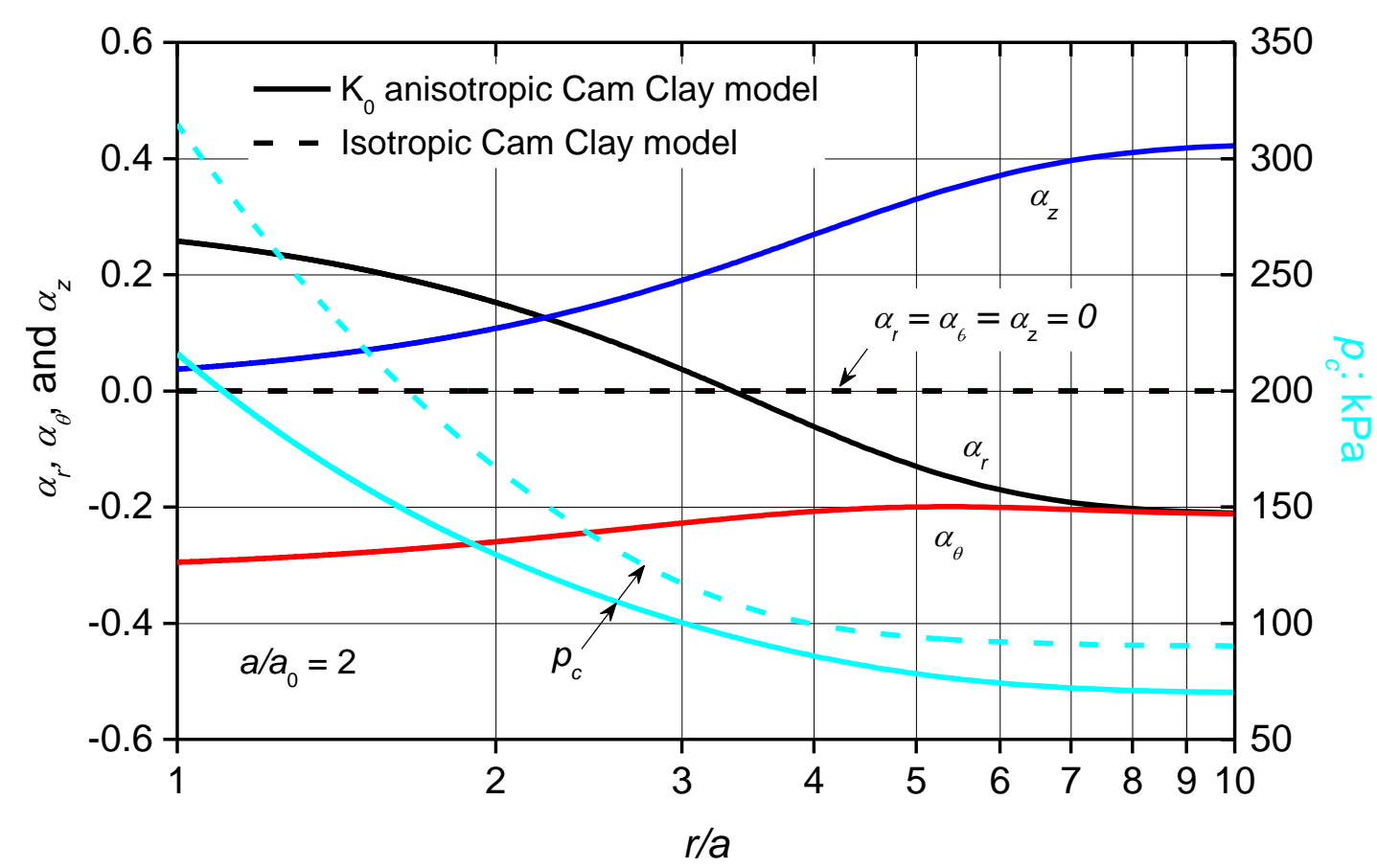

Fig. 8. Influences of $K_{0}$ consolidation anisotropy on the distributions of $\alpha_{r}, \alpha_{\theta}, \alpha_{z}$ and $p_{c}$ around the cavity [solid and dashed lines represent anisotropic and isotropic solutions, respectively] 


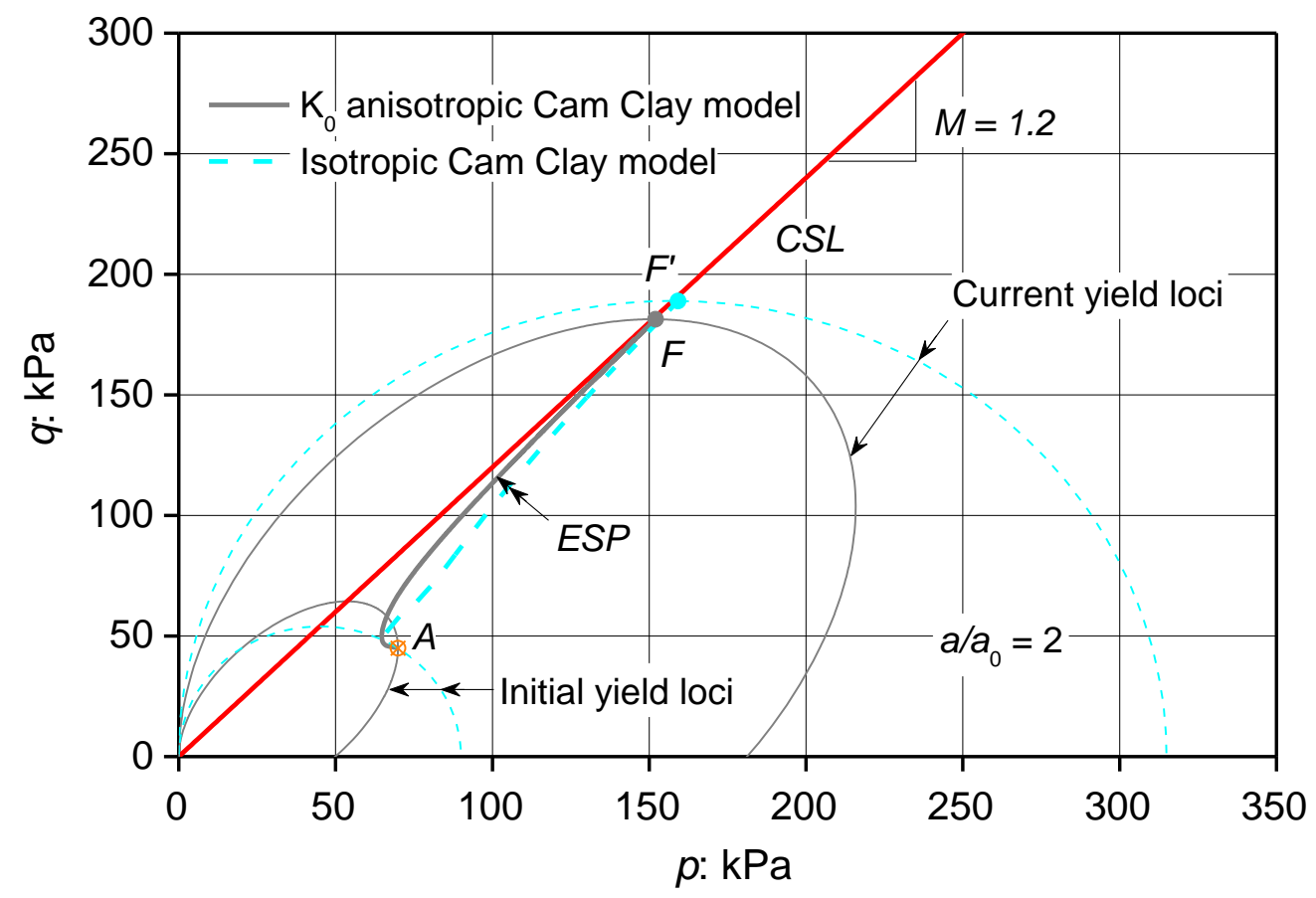

Fig. 9. Influences of $K_{0}$ consolidation anisotropy on the p-q stress path 


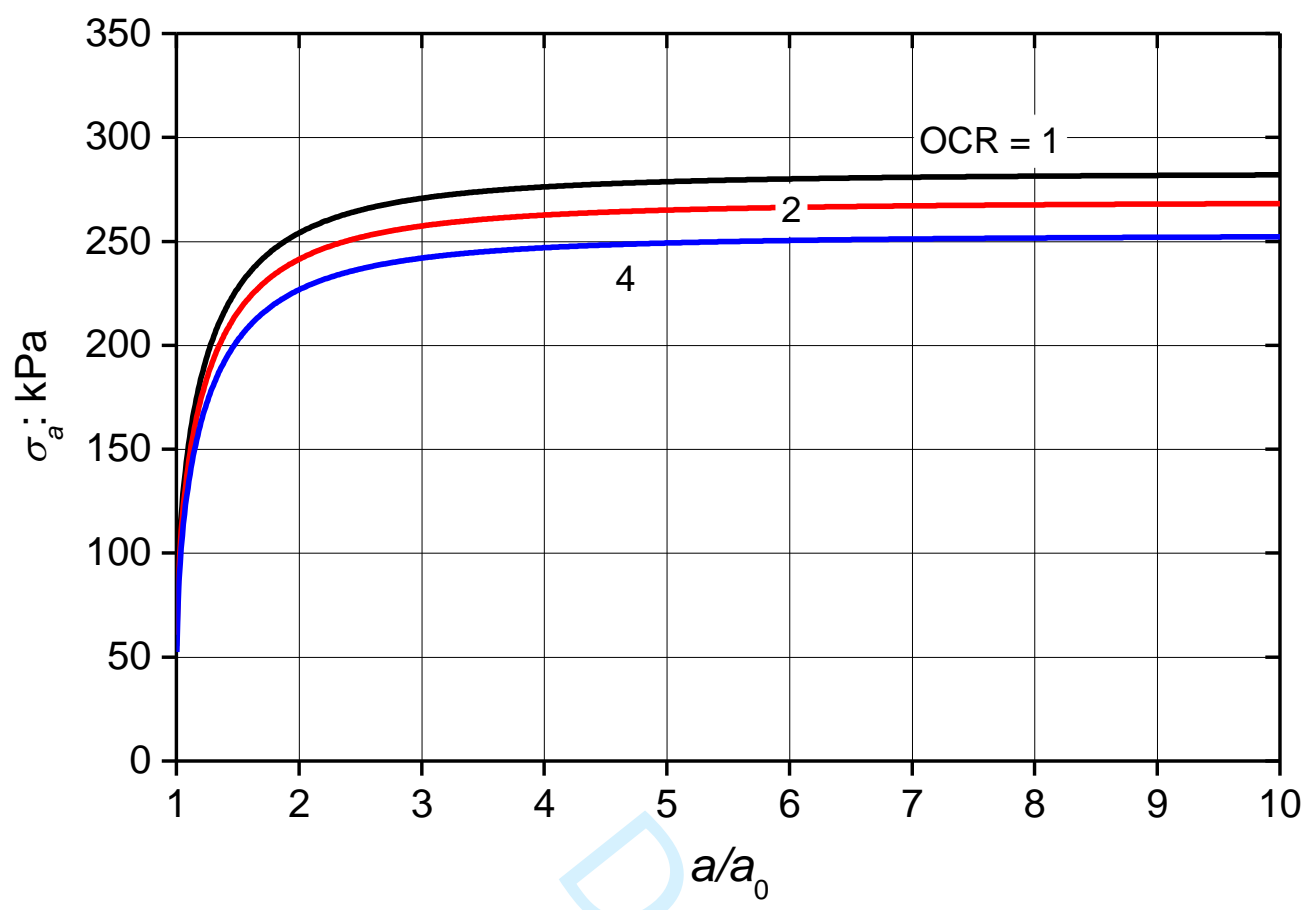

Fig. 10. Influences of overconsolidation ratio on the cavity expansion curve 

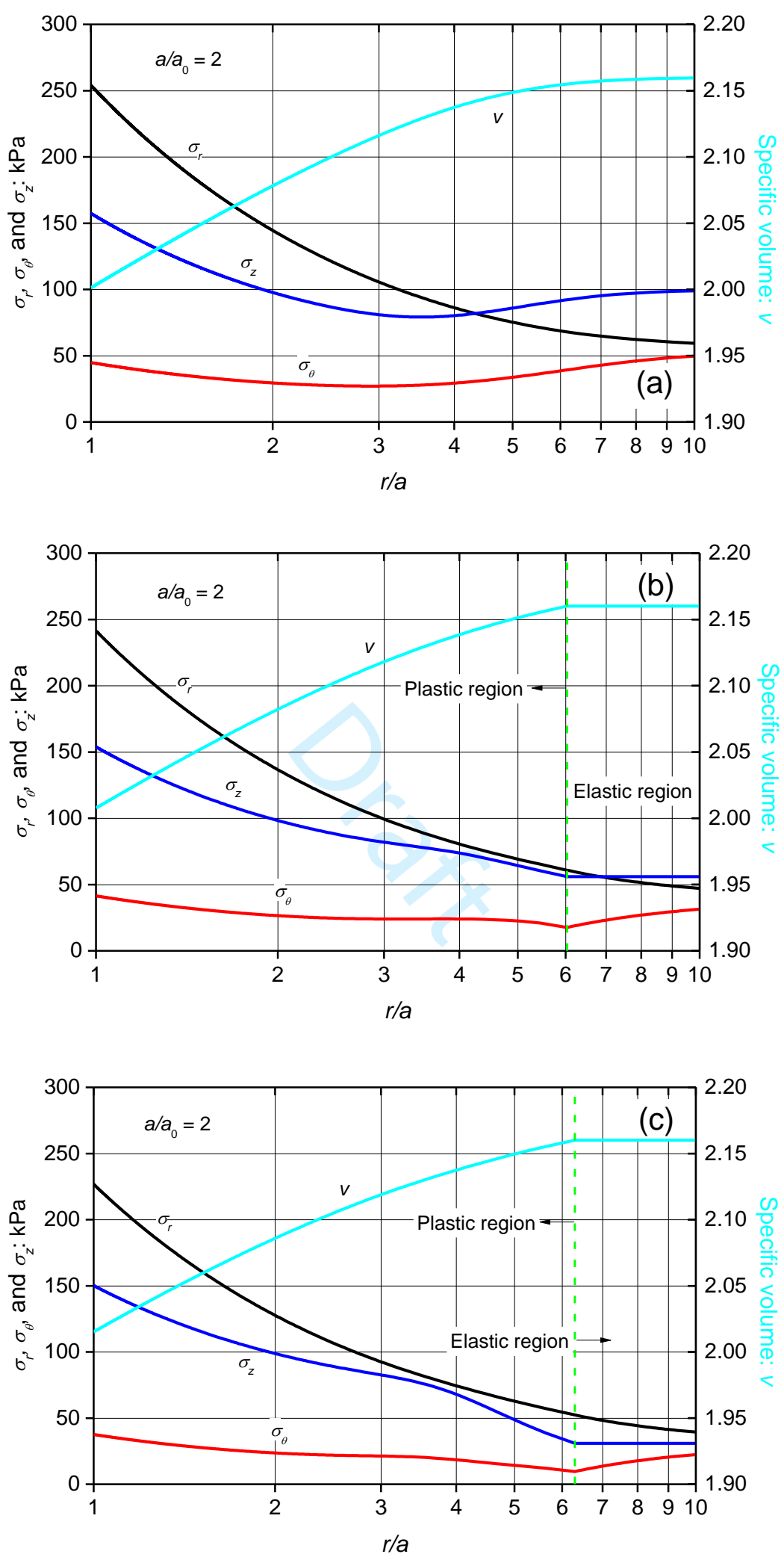

Fig. 11. Distributions of radial, tangential, vertical stresses and specific volume along the radial distance: (a) $\mathrm{OCR}=1$; (b) $\mathrm{OCR}=2$; and (c) $\mathrm{OCR}=4$ 

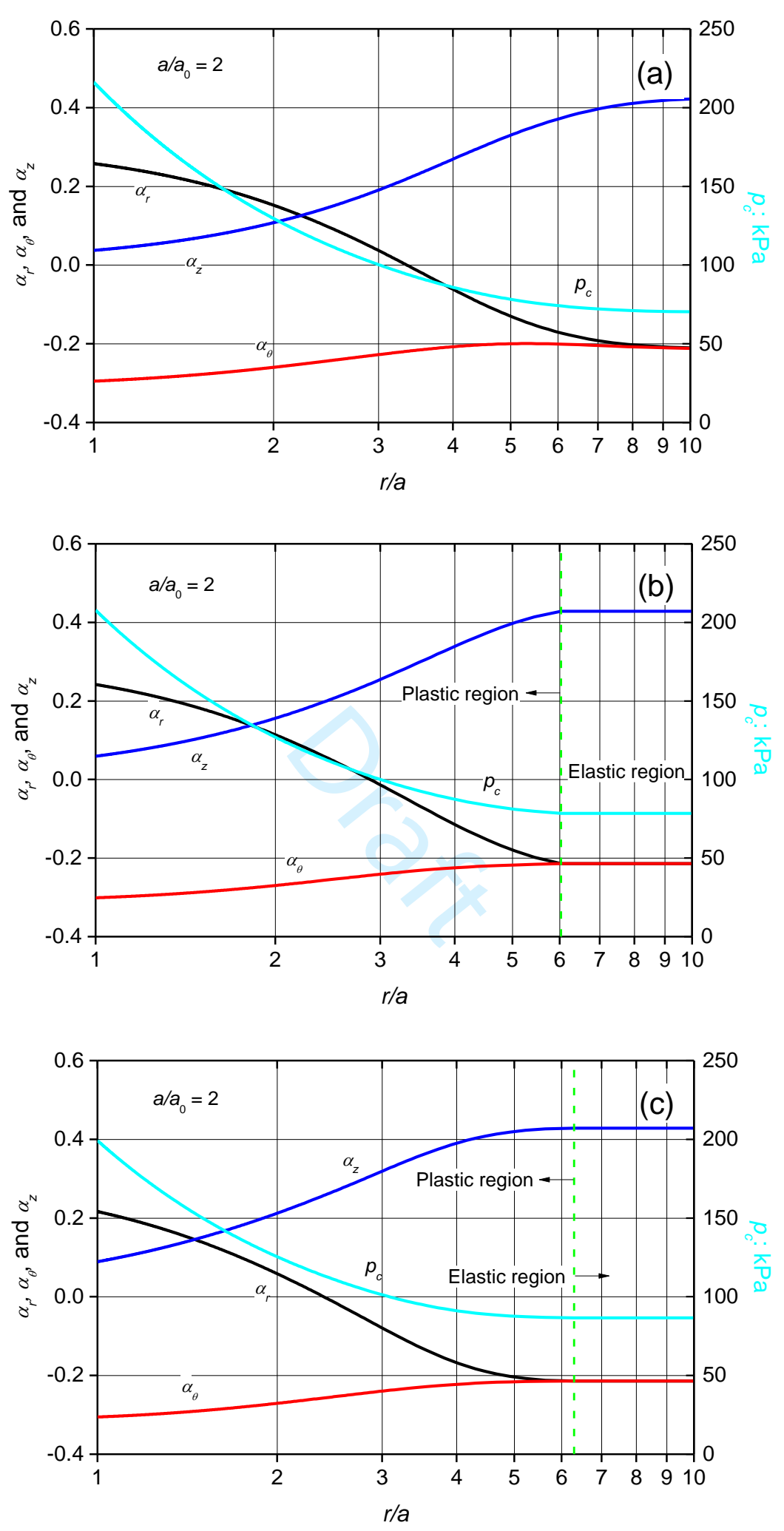

Fig. 12. Distributions of hardening parameter $p_{c}$ and anisotropic variables $\alpha_{r}, \alpha_{\theta}, \alpha_{z}$ along the radial distance: (a) $\mathrm{OCR}=1$; (b) $\mathrm{OCR}=2$; and (c) $\mathrm{OCR}=4$ 

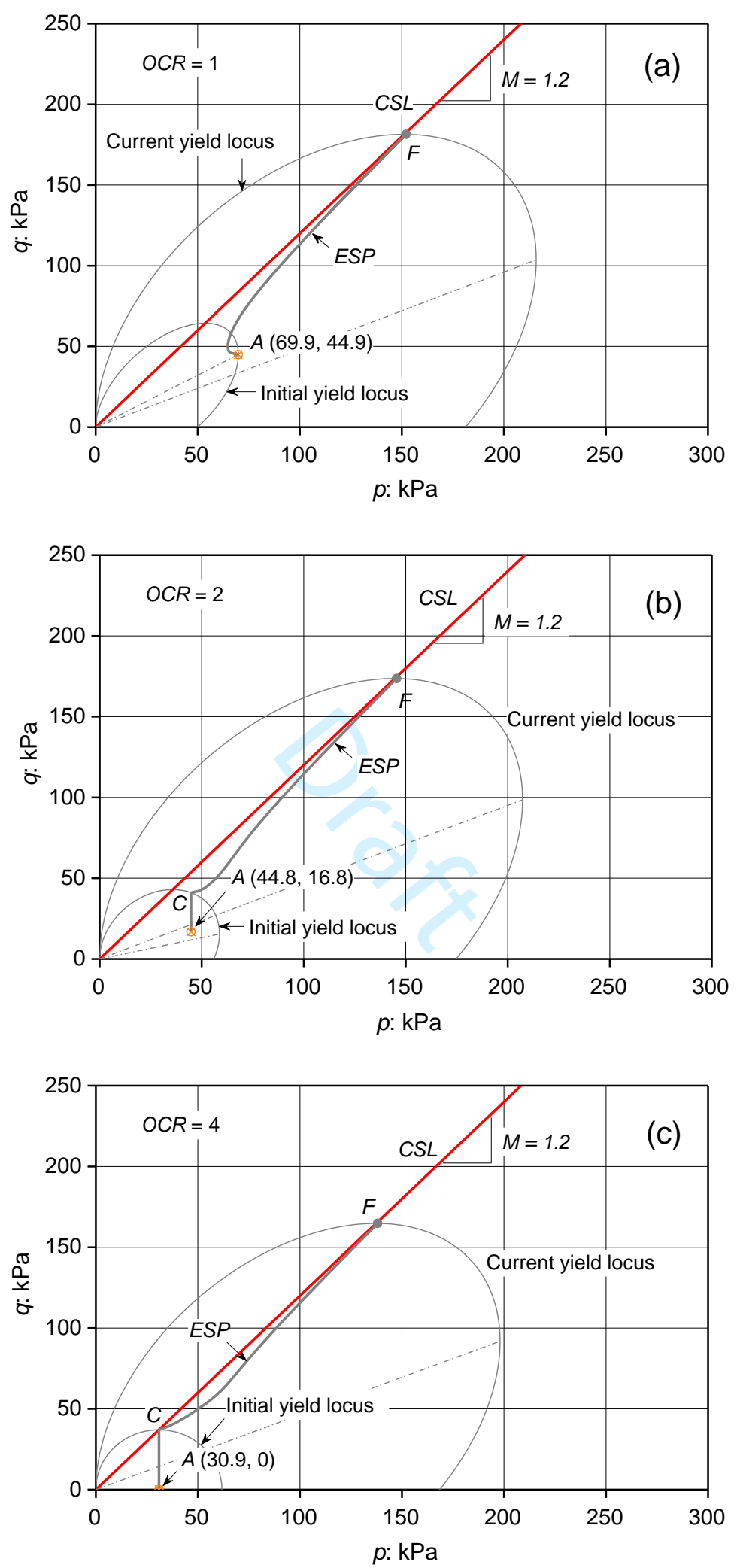

Fig. 13. Stress path followed by a soil particle at cavity wall in p-q plane and deviatoric plane: (a) and (d) $O C R=1$; (b) and (e) $O C R=2$; (c) and (f) $O C R=4$ 

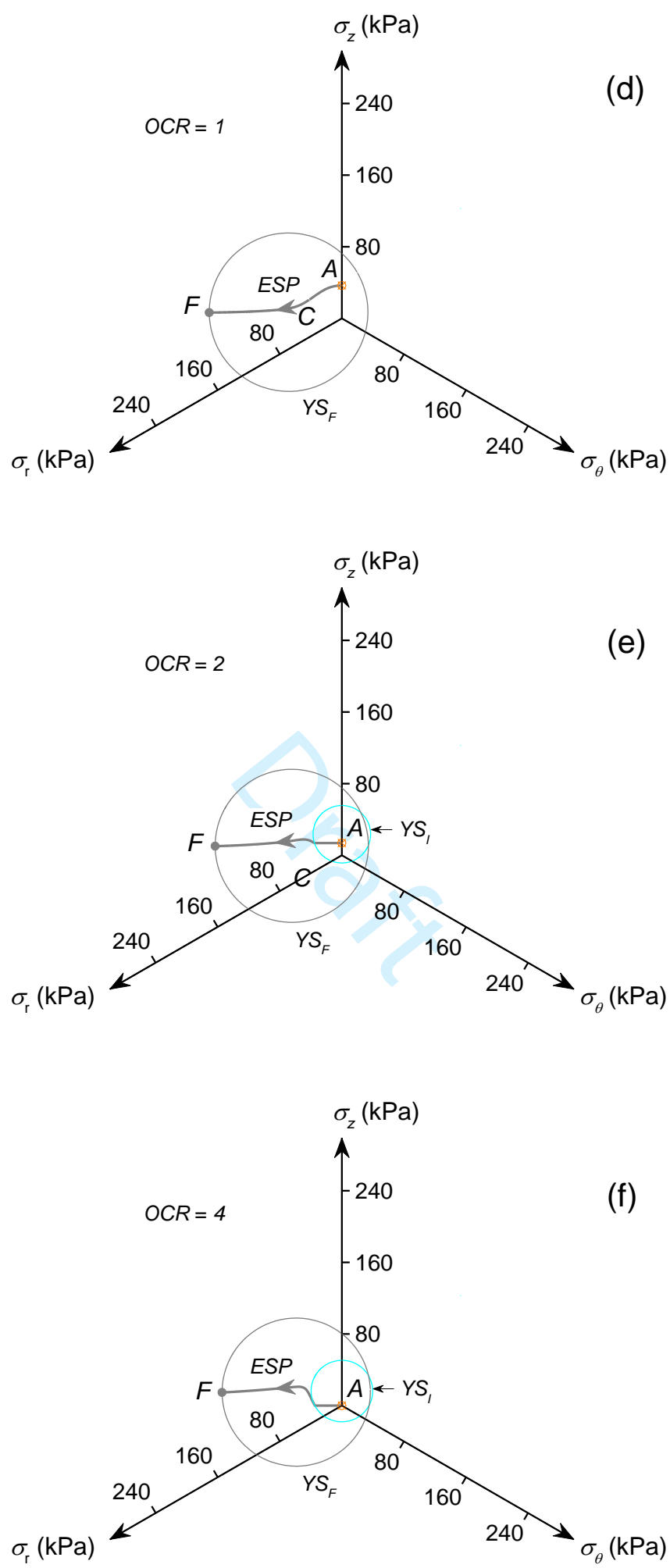

Fig. 13. (continued) Stress path followed by a soil particle at cavity wall in p-q plane and deviatoric plane: (a) and (d) $O C R=1$; (b) and (e) $O C R=2$; (c) and (f) $O C R=4$ 